ULB-TH/09-28

\title{
Aspects of the BMS/CFT correspondence
}

\author{
Glenn Barnich ${ }^{a}$ and Cédric Troessaert ${ }^{b}$ \\ Physique Théorique et Mathématique \\ Université Libre de Bruxelles \\ and \\ International Solvay Institutes \\ Campus Plaine C.P. 231, B-1050 Bruxelles, Belgium
}

\begin{abstract}
After a review of symmetries and classical solutions involved in the $\mathrm{AdS}_{3} / \mathrm{CFT}_{2}$ correspondence, we apply a similar analysis to asymptotically flat spacetimes at null infinity in 3 and 4 dimensions. In the spirit of two dimensional conformal field theory, the symmetry algebra of asymptotically flat spacetimes at null infinity in 4 dimensions is taken to be the semi-direct sum of supertranslations with infinitesimal local conformal transformations and not, as usually done, with the Lorentz algebra. As a first application, we derive how the symmetry algebra is realized on solution space. In particular, we work out the behavior of Bondi's news tensor, mass and angular momentum aspects under local conformal transformations.
\end{abstract}

${ }^{a}$ Research Director of the Fund for Scientific Research-FNRS. E-mail: gbarnich@ulb.ac.be

${ }^{b}$ Research Fellow of the Fund for Scientific Research-FNRS. E-mail: ctroessa@ulb.ac.be 


\section{Contents}

1 Introduction 3

2 Asymptotically $\mathrm{AdS}_{3}$ spacetimes in Fefferman-Graham form

2.1 Asymptotic symmetries . . . . . . . . . . . . . . . . . 5

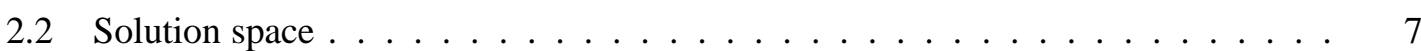

2.3 Conformal properties of solution space . . . . . . . . . . . . 9

2.4 Centrally extended surface charge algebra $\ldots \ldots \ldots \ldots$

$3 \mathrm{BMS}_{3} / \mathrm{CFT}_{1}$ correspondence 12

3.1 Asymptotic symmetries . . . . . . . . . . . . . . . 13

3.2 Solution space . . . . . . . . . . . . . . . . 15

3.3 Conformal properties of solution space . . . . . . . . . . . 17

3.4 Centrally extended surface charge algebra . . . . . . . . . . . . 17

$4 \mathrm{BMS}_{4} / \mathrm{CFT}_{2}$ correspondence 18

4.1 Asymptotically flat 4 -d spacetimes at null infinity . . . . . . . . . . 18

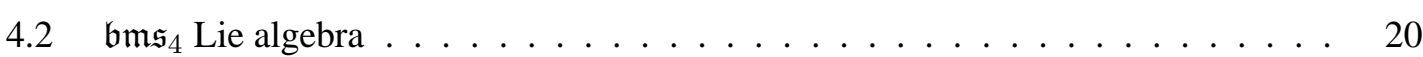

4.3 Solution space . . . . . . . . . . . . . . . . . . 24

4.4 Realization of $\mathfrak{b m s}_{4}$ on solution space $\ldots \ldots \ldots \ldots \ldots \ldots$

5 Conclusion and outlook 35

Acknowledgments 


\section{Introduction}

When studying dualities between string or gravitational theories in anti-de Sitter backgrounds and conformal theories on their boundaries [1], the 3-dimensional case is especially interesting. Indeed, the symmetries of asymptotically $A d S$ spacetimes [2, 3, 4] provide a representation of the algebra of conformal Killing vectors of the flat boundary metric. In 3 spacetime dimensions, this algebra is infinite-dimensional and powerful techniques of 2-dimensional conformal field theory are available.

Historically, the first example where the asymptotic symmetry group is enhanced with respect to the isometry group of the background metric and becomes infinite-dimensional is the one of asymptotically flat 4-dimensional spacetimes at null infinity [5, 6, 7]. In this case, the induced metric is 2-dimensional because the boundary is a null surface. The asymptotic symmetry group of non singular transformations is the well-known BondiMetzner-Sachs group. It consists of the semi-direct product of the group of globally defined conformal transformations of the unit 2-sphere, which is isomorphic to the orthochronous homogeneous Lorentz group, times the infinite-dimensional abelian normal subgroup of so-called supertranslations.

There is a further enhancement when one focuses on infinitesimal transformations and does not require the associated finite transformations to be globally well-defined. The symmetry algebra is then the semi-direct sum of the infinitesimal local conformal transformations of the 2-sphere with the abelian ideal of supertranslations, and now both factors are infinite-dimensional [8].

A first hint on what the symmetry algebra of asymptotically flat spacetimes at null infinity might be, independently of precise fall-off conditions on the metric, can be obtained by solving the Killing equations to leading order. This has been done in [9] in all dimensions greater than 3. In 4 dimensions, the infinite-dimensional nature of the conformal factor of the $\mathfrak{b m s}_{4}$ algebra has been emphasized. In 3 dimensions, the asymptotic symmetry algebra $\mathfrak{b m s}_{3}$, originally derived in [10, 11], has been recovered. The algebra of surface charges has been shown to provide a centrally extended representation of $\mathfrak{b m s}_{3}$ which has been related by a contraction, similiar to that from $\mathfrak{s o}(2,2)$ to iso $(2,1)$, to the centrally extended Poisson bracket algebra of surface charges of asymptotically anti-de Sitter spacetimes in 3 dimensions.

The aim of the present work is to reconsider from the point of view of local conformal transformations the 4-dimensional case which is, in some sense at least, of direct physical relevance. In particular, we provide a detailed derivation of the natural generalization of the $\mathfrak{b m s}_{4}$ algebra discussed above. No modification of well studied boundary conditions is needed and the transformations are carefully distinguished from conformal rescalings.

A major motivation for our investigation comes from Strominger's derivation [12] 
of the Bekenstein-Hawking entropy for black holes that have a near horizon geometry that is locally $A d S_{3}$ by using the Brown-Henneaux analysis of the surface charge algebra of asymptotically anti-de Sitter spacetimes at spatial infinity. More recently, a similar analysis has been used to derive the Bekenstein-Hawking entropy of an extreme 4-dimensional Kerr black hole [13]. One of our hopes is to make progress along these lines in the non extreme case, either directly from an analysis at null infinity or by making a similar analysis at the horizon, as discussed previously for instance in $[14,15,16,17,18,19,20,21,22,23,24,25,26]$.

Related work includes [27, 28] on asymptotic quantization where for instance the implications of supertranslations for the gravitational $S$-matrix have been discussed. Asymptotically flat spacetimes at null infinity in higher spacetime dimensions have been investigated for instance in [29, 30, 31, 32], while various aspects of holography in 4 dimensions have been studied in some details in [33, 34, 35, 36, 37, 38, 39]. In particular, a symmetry algebra of the kind that we derive and study here has been conjectured in [40].

This paper is organized as follows. Asymptotically $A d S_{3}$ spacetimes in FeffermanGraham form are briefly reviewed in the next section in order to illustrate the approach adopted for the asymptotically flat case in a well-studied situation. In order to be selfcontained, we include details of the computations. The section is based on results originally derived and discussed for instance in [41, 3, 42, 43, 44, 45, 46, 47, 48, 49, 50]: in particular, for asymptotically $A d S_{3}$ spacetimes in the sense of Fefferman-Graham, the gauge is completely fixed as all subleading orders of the asymptotic Killing vectors are uniquely determined; furthermore, the general solution of the equations of motion can be written down in closed form for an arbitary boundary metric. We then compute how the local conformal algebra in 2 dimensions is realized on solution space and analyze how this realization is probed by a covariant version of the Brown-Henneaux charge algebra.

A novel result in this context concerns the representation of the local conformal algebra and its abelian extension by conformal rescalings in terms of spacetime vectors equipped with a new, modified Dirac-type Lie bracket taking into account the metric dependence of the asymptotic Killing vectors due to the gauge fixing.

In section 3 , we re-analyze asymptotically flat spacetimes at null infinity in 3 dimensions. We start by rederiving their symmetries in the form suggested by the analysis of the 4 dimensional case by Sachs and by the Fefferman-Graham analysis of asymptotically $A d S$ spacetimes in 3 dimensions. In particular, we discuss the extended symmetry algebra and its representation in terms of spacetime vectors equipped with the modified Lie bracket. Furthermore, we provide the general solution to the equations of motions. We then analyze how $\mathfrak{b m s}_{3}$ is realized on solution space and how this realization is probed by the centrally extended covariant surface charge algebra.

In the main section 4 on asymptotically flat spacetimes at null infinity in 4 spacetime 
dimensions, we derive the asymptotic algebra and prove the main result on local conformal transformations announced in [8]. In order to do so, we follow closely the original analysis of Bondi, Metzner and Sachs [5, 6, 7], but as suggested by the geometrical analysis of Penrose [51], we allow for an arbitrary conformal factor in the boundary metric. More precisely, by allowing meromorphic functions on the Riemann sphere, we show that the asymptotic symmetry algebra involves instead of the Lorentz algebra two copies of the Witt algebra that act in a natural way on the abelian ideal of supertranslations. By restricting ourselves to exact isometries of Minkowski spacetime, we identify the Poincaré subalgebra of $\mathfrak{b m s}_{4}$. We show how the asymptotic symmetry algebra is represented by the spacetime vectors equipped with the modified Lie bracket and generalize these results to the extended algebra including conformal rescalings of the boundary metric.

We then turn to a detailed discussion of solution space by mostly following [52, 53]. Under appropriate assumptions, the arbitrary functions which arise as integration constants in the general solution to the equations of motion are identified. They involve in particular the so-called news tensor together with the mass and angular momentum aspects. We include a discussion of the leading logarithmic term and generalize existing results by allowing the conformal factor to explicitly depend on time.

The second main result consists in deriving the transformation properties under the asymptotic symmetry algebra $\mathfrak{b m s}_{4}$ of some of the fields characterizing the solutions. In particular, conformal dimensions and central charges under local conformal transformations of the news tensor, the mass and angular momentum aspects are worked out.

\section{Asymptotically $\mathrm{AdS}_{3}$ spacetimes in Fefferman-Graham form}

The Fefferman-Graham form for the line element of a 3 dimensional asymptotically antide Sitter spacetime is

$$
d s^{2}=\frac{l^{2}}{r^{2}} d r^{2}+g_{A B}\left(r, x^{C}\right) d x^{A} d x^{B},
$$

with $g_{A B}=r^{2} \bar{\gamma}_{A B}\left(x^{C}\right)+O(1)$, where $\bar{\gamma}_{A B}$ is a conformally flat 2-dimensional metric. For explicit computations we will sometimes choose the parametrization $\bar{\gamma}_{A B}=e^{2 \varphi} \eta_{A B}$ with $\varphi\left(x^{C}\right)$ and $\eta_{A B}$ the flat metric on the cylinder, $\eta_{A B} d x^{A} d x^{B}=-d \tau^{2}+d \phi^{2}, \tau=\frac{1}{l} t$.

\subsection{Asymptotic symmetries}

The transformations leaving this form of the metric invariant are generated by vector fields satisfying

$$
\mathcal{L}_{\xi} g_{r r}=0=\mathcal{L}_{\xi} g_{r A}, \quad \mathcal{L}_{\xi} g_{A B}=O(1),
$$


which implies

$$
\left\{\begin{array}{l}
\xi^{r}=-\frac{1}{2} \psi r, \\
\xi^{A}=Y^{A}+I^{A}, \quad I^{A}=-\frac{l^{2}}{2} \partial_{B} \psi \int_{r}^{\infty} \frac{d r^{\prime}}{r^{\prime}} g^{A B}=-\frac{l^{2}}{4 r^{2}} \bar{\gamma}^{A B} \partial_{B} \psi+O\left(r^{-4}\right),
\end{array}\right.
$$

where $Y^{A}$ is a conformal Killing vector of $\bar{\gamma}_{A B}$, and thus of $\eta_{A B}$, while $\psi=\bar{D}_{A} Y^{A}$ is the conformal factor.

Indeed, the inverse to metric (2.1) is

$$
g^{\mu \nu}=\left(\begin{array}{cc}
\frac{r^{2}}{l^{2}} & 0 \\
0 & g^{A B}
\end{array}\right)
$$

where $g^{A B} g_{B C}=\delta_{C}^{A}$. From $\mathcal{L}_{\xi} g_{r r}=0$, we find $\xi^{r}=A r$ for some $A\left(x^{C}\right)$. From $\mathcal{L}_{\xi} g_{r A}=0$ we find $\partial_{r} \xi^{A}=-g^{A B} \frac{l^{2}}{r} \partial_{B} A$ so that $\xi^{A}=Y^{A}+I^{A}$ for some $Y^{A}\left(x^{C}\right)$ and where $I^{A}=l^{2} \partial_{B} A \int_{r}^{\infty} d r^{\prime} g^{A B} r^{\prime-1}$. Finally, $\mathcal{L}_{\xi} g_{A B}=O(1)$ requires $Y^{A}$ to be a conformal Killing vector of $\bar{\gamma}_{A B}$ and $A=-\frac{1}{2} \psi$.

Let $\widehat{Y}^{A}=\left[Y_{1}, Y_{2}\right]^{A}, \widehat{\psi}=\bar{D}_{A} \widehat{Y}^{A}$, denote by $\delta_{\xi_{1}}^{g} \xi_{2}^{\mu}$ the change induced in $\xi_{2}^{\mu}(g)$ due to the variation $\delta_{\xi_{1}}^{g} g_{\mu \nu}=\mathcal{L}_{\xi_{1}} g_{\mu \nu}$ and define

$$
\left[\xi_{1}, \xi_{2}\right]_{M}^{\mu}=\left[\xi_{1}, \xi_{2}\right]^{\mu}-\delta_{\xi_{1}}^{g} \xi_{2}^{\mu}+\delta_{\xi_{2}}^{g} \xi_{1}^{\mu} .
$$

For vectors $\xi_{1}, \xi_{2}$ given in (2.3), we have

$$
\left[\xi_{1}, \xi_{2}\right]_{M}^{r}=-\frac{1}{2} \widehat{\psi} r, \quad\left[\xi_{1}, \xi_{2}\right]_{M}^{A}=\widehat{Y}^{A}+\widehat{I}^{A}
$$

where $\widehat{I}^{A}$ denotes $I^{A}$ with $\psi$ replaced by $\widehat{\psi}$.

Indeed, for the $r$ component, we have $\delta_{\xi_{1}}^{g} \xi_{2}^{r}=0$ and the result follows by direct computation of the Lie bracket. Similarily, $\lim _{r \rightarrow \infty}\left[\xi_{1}, \xi_{2}\right]_{M}^{A}=\widehat{Y}^{A}$. Finally, using $\partial_{r} \xi^{r}=\frac{1}{r} \xi^{r}$ and $\partial_{r} \xi^{A}=-\frac{l^{2}}{r^{2}} \partial_{B} \xi^{r} g^{B A}$ a straightforward computation shows that $\partial_{r}\left(\left[\xi_{1}, \xi_{2}\right]_{M}^{A}\right)=$ $-\frac{l^{2}}{r^{2}} \partial_{B}\left(\left[\xi_{1}, \xi_{2}\right]_{M}^{r}\right) g^{B A}$, which gives the result. It thus follows that on an asymptotically anti-de Sitter spacetime in the sense of Fefferman-Graham (solving or not Einstein's equations with cosmological constant):

The spacetime vectors (2.3) equipped with the bracket $[\cdot, \cdot]_{M}$ form a faithful representation of the conformal algebra.

By conformal algebra, we mean here the direct sum of 2 copies of the Witt algebra. Furthermore, since $\delta_{\xi_{1}}^{g} \xi_{2}^{r}=0, \delta_{\xi_{1}}^{g} \xi_{2}^{A}=O\left(r^{-4}\right)$, it follows that these vectors form a representation of the conformal algebra only up to terms of order $O\left(r^{-4}\right)$ when equipped with the standard Lie bracket.

In terms of light-cone coordinates, $x^{ \pm}=\tau \pm \phi, 2 \partial_{ \pm}=\frac{\partial}{\partial \tau} \pm \frac{\partial}{\partial \phi}$, we have $\bar{\gamma}_{A B} d x^{A} d x^{B}=$ $-e^{2 \varphi} d x^{+} d x^{-}$, and if,

$$
Y^{ \pm}\left(x^{ \pm}\right) \partial_{ \pm}=\sum_{n \in \mathbf{Z}} c_{ \pm}^{n} l_{n}^{ \pm}, \quad l_{n}^{ \pm}=-\left(x^{ \pm}\right)^{n+1} \partial_{ \pm},
$$


the algebra in terms of the basis vectors $l_{n}^{ \pm}$reads

$$
\left[l_{m}^{ \pm}, l_{n}^{ \pm}\right]=(m-n) l_{m}^{ \pm}, \quad\left[l_{m}^{ \pm}, l_{n}^{\mp}\right]=0 .
$$

More generally, one can also consider the transformations that leave the FeffermanGraham form of the metric invariant up to a Weyl rescaling of the boundary metric $\bar{\gamma}_{A B}$. They are generated by spacetime vectors such that

$$
\mathcal{L}_{\xi} g_{r r}=0=\mathcal{L}_{\xi} g_{r A}, \quad \mathcal{L}_{\xi} g_{A B}=2 \omega g_{A B}+O(1) .
$$

It is then straightforward to see that the general solution is given by the vectors (2.3), where $\psi$ is replaced by $\widetilde{\psi}=\psi-2 \omega$. When equipped with the modified Lie bracket $[\cdot, \cdot]_{M}$ these vectors now form a faithful representation of the extension of the two dimensional conformal algebra defined by elements $(Y, \omega)$ and the Lie bracket $(\widehat{Y}, \widehat{\omega})=$ $\left[\left(Y_{1}, \omega_{1}\right),\left(Y_{2}, \omega_{2}\right)\right]$,

$$
\widehat{Y}^{A}=Y_{1}^{B} \partial_{B} Y_{2}^{A}-Y_{2}^{B} \partial_{B} Y_{1}^{A}, \quad \widehat{\omega}=0,
$$

with $\omega\left(x^{C}\right)$ arbitrary and $Y^{A}$ conformal Killing vectors of $\bar{\gamma}_{A B}$ and thus also of $\eta_{A B}$. The asymptotic symmetry algebra is then the direct sum of the abelian ideal of elements of the form $(0, \omega)$ and of 2 copies of the Witt algebra.

Indeed, we have $\lim _{r \rightarrow \infty}\left(\frac{1}{r}\left[\xi_{1}, \xi_{2}\right]_{M}^{r}\right)=-\frac{1}{2} Y_{1}^{A} \partial_{A} \widetilde{\psi}_{2}+\partial_{C} \omega_{1} Y_{2}^{C}+(1 \leftrightarrow 2)=-\frac{1}{2} \widehat{\psi}$ and $\partial_{r}\left(\frac{1}{r}\left[\xi_{1}, \xi_{2}\right]_{M}^{r}\right)=0$, while the proof for the $A$-component is unchanged.

\subsection{Solution space}

Let us now start with an arbitrary metric of the form (2.1), without any assumptions on the behavior in $r$ and let $k_{B}^{A}=\frac{1}{2} g^{A C} g_{C B, r}$. One can then define $K_{B}^{A}$ through the relation $k_{B}^{A}=\frac{1}{r} \delta_{B}^{A}+\frac{1}{r^{3}} K_{B}^{A}$. We have

$$
\begin{gathered}
\Gamma_{r r}^{r}=-\frac{1}{r}, \quad \Gamma_{r A}^{r}=0, \quad \Gamma_{r r}^{A}=0, \\
\Gamma_{A B}^{r}=-\frac{r^{2}}{l^{2}} k_{A B}, \quad \Gamma_{r B}^{A}=k_{B}^{A}, \quad \Gamma_{B C}^{A}={ }^{(2)} \Gamma_{B C}^{A},
\end{gathered}
$$

where ${ }^{(2)} \Gamma_{B C}^{A}$ denotes the Christoffel symbol associated to the 2-dimensional metric $g_{A B}$, which is used to lower indices on $k_{B}^{A}$. If $K^{T A}{ }_{B}$ denotes the traceless part of $K_{B}^{A}$, the equations of motion are organized as follows

$$
\begin{gathered}
g^{A B} G_{A B}-\frac{2}{l^{2}}=0 \Longleftrightarrow \partial_{r} K=-r^{-3}\left(\frac{1}{2} K^{2}+K_{B}^{T_{B}^{A}} K_{A}^{T_{A}^{B}}\right), \\
G_{A B}-\frac{1}{2} g_{A B} g^{C D} G_{C D}=0 \Longleftrightarrow \partial_{r} K_{B}^{T_{B}^{A}}=-r^{-3} K K_{B}^{T_{B}^{A},} \\
G_{r A} \equiv r^{-3}\left({ }^{(2)} D_{B} K_{A}^{B}-\partial_{A} K\right)=0, \\
G_{r r}-\frac{1}{l^{2}} g_{r r} \equiv \frac{1}{2}\left[r^{-6}\left(\frac{1}{2} K^{2}-K_{B}^{T_{B}^{A}} K_{A}^{T^{B}}\right)+2 r^{-4} K-\frac{l^{2}}{r^{2}}{ }^{(2)} R\right]=0 .
\end{gathered}
$$


Combining the Bianchi identities $2\left(\sqrt{-g} G_{\alpha}^{\beta}\right)_{, \beta}+\sqrt{-g} G_{\beta \gamma} g^{\beta \gamma}{ }_{, \alpha} \equiv 0$ with the covariant constancy of the metric, we get the identities

$$
\begin{aligned}
& 2\left(\frac{r}{l} \sqrt{\left.\right|^{(2)} g \mid} G_{r A}\right)_{, r}+2\left(\frac{l}{r} \sqrt{\left.\right|^{(2)} g \mid} g^{B C}\left[G_{C A}-\frac{1}{l^{2}} g_{C A}\right]\right)_{, B}+ \\
& +\frac{l}{r} \sqrt{\left|{ }^{(2)} g\right|}\left(G_{B C}-\frac{1}{l^{2}} g_{B C}\right) g_{, A}^{B C} \equiv 0, \\
& \left(\frac{r}{l} \sqrt{\left|{ }^{(2)} g\right|}\left[G_{r r}-\frac{1}{r^{2}}\right]\right)_{, r}+\left(\frac{l}{r} \sqrt{\left.\right|^{(2)} g \mid} g^{B A} G_{A r}\right)_{, B}+ \\
& +\frac{1}{l} \sqrt{\left.\right|^{(2)} g \mid}\left[G_{r r}-\frac{1}{r^{2}}\right]-\frac{l}{r} \sqrt{\left|{ }^{(2)} g\right|}\left(G_{A B}-\frac{1}{l^{2}} g_{A B}\right) k^{A B} \equiv 0 .
\end{aligned}
$$

To solve the equations of motion, we first contract (2.10) with $K_{A}^{T^{B}}$, which gives

$$
\partial_{r}\left(K_{B}^{T_{B}^{A}} K_{A}^{T}{ }_{A}^{B}\right)=-2 r^{-3} K K_{B}^{T}{ }_{B}^{A} K_{A}^{T} .
$$

If we assume $K_{B}^{T}{ }_{B}^{A} K_{A}^{T}=\frac{1}{2} \mathcal{K}^{2}$, we can take the sum and difference with (2.9) to get

$$
\partial_{r}(K+\mathcal{K})=-\frac{1}{2} r^{-3}(K+\mathcal{K})^{2}, \quad \partial_{r}(K-\mathcal{K})=-\frac{1}{2} r^{-3}(K-\mathcal{K})^{2},
$$

which can be solved in terms of 2 integration "constants" $C\left(x^{B}\right), D\left(x^{B}\right)$

$$
K=-\frac{1}{C+\frac{1}{2} r^{-2}}-\frac{1}{D+\frac{1}{2} r^{-2}}, \quad K_{B}^{T}{ }_{B}^{A} K_{A}^{T}=\frac{(D-C)^{2}}{2\left(C+\frac{1}{2} r^{-2}\right)^{2}\left(D+\frac{1}{2} r^{-2}\right)^{2}} .
$$

When used in (2.10), we find

$$
K_{B}^{T_{B}^{A}}=A_{B}^{T_{B}^{A}}\left(\frac{1}{C+\frac{1}{2} r^{-2}}-\frac{1}{D+\frac{1}{2} r^{-2}}\right), \quad A_{B}^{T_{B}^{A}} A_{A}^{T^{B}}=\frac{1}{2},
$$

and can now reconstruct the metric from the equation $\partial_{r} g_{A B}=2 g_{A C} k_{B}^{C}$. Defining $\Theta=$ $\frac{1}{D}+\frac{1}{C}, \Omega=\frac{1}{D}-\frac{1}{C}$, we get

$$
g_{A B}=r^{2} \bar{\gamma}_{A B}\left[1+\frac{1}{2 r^{2}} \Theta+\frac{1}{16 r^{4}}\left(\Theta^{2}+\Omega^{2}\right)\right]+A_{A B}^{T}\left[\Omega+\frac{1}{4 r^{2}} \Theta \Omega\right],
$$

where $\bar{\gamma}_{A B}$ are additional integration constants, restricted by the condition that $\bar{\gamma}_{A B}$ is symmetric, of signature -1 . The index on $A_{B}^{T A}$ is lowered with $\bar{\gamma}_{A B}$, with $A_{A B}^{T}$ requested to be symmetric. It follows that $A_{B}^{T_{B}^{A}}$ contains only 1 additional independent integration constant. Writing $g_{A B}=r^{2} \bar{\gamma}_{A B}+\gamma_{A B}$, with $\gamma_{A B}=\widehat{\gamma}_{A B}+o\left(r^{0}\right)$, we have $K_{B}^{A}=$ $-\widehat{\gamma}_{B}^{A}+o\left(r^{0}\right)$ where the index on $\widehat{\gamma}_{B}^{A}$ has been lifted with $\bar{\gamma}^{A B}$, the inverse of $\bar{\gamma}_{A B}$.

When (2.9) and (2.10) are satisfied, the Bianchi identity (2.13) implies that $r \sqrt{\left|{ }^{(2)} g\right|} G_{r A}$ does not depend on $r$. The equation of motion (2.11) then reduces to the condition

$$
\bar{D}_{B} \widehat{\gamma}_{A}^{B}-\partial_{A} \widehat{\gamma}=0
$$


where $\widehat{\gamma}=\widehat{\gamma}_{A}^{A}$. When this condition holds in addition to (2.9) and (2.10), the remaining Bianchi identity (2.14) implies that $r^{2} \sqrt{\left.\right|^{(2)} g \mid}\left[G_{r r}-\frac{1}{r^{2}}\right]$ does not depend on $r$. The equation of motion (2.12) then reduces to the condition

$$
\widehat{\gamma}=-\frac{l^{2}}{2} \bar{R}
$$

and also from the leading contribution to $K$ that $\Theta=-\frac{l^{2}}{2} \bar{R}$. The constraint (2.16) then becomes

$$
\bar{D}_{B} \widehat{\gamma}_{A}^{T B}=-\frac{l^{2}}{4} \partial_{A} \bar{R}
$$

To solve this equation, one uses light-cone coordinates, $x^{ \pm}=\tau \pm \phi, 2 \partial_{ \pm}=\frac{\partial}{\partial \tau} \pm \frac{\partial}{\partial \phi}$ and the explicit parameterization $\bar{\gamma}_{A B} d x^{A} d x^{B}=-e^{2 \varphi} d x^{+} d x^{-}$. This gives

$$
\widehat{\gamma}=-4 l^{2} e^{-2 \varphi} \partial_{+} \partial_{-} \varphi \Longleftrightarrow \widehat{\gamma}_{+-}=l^{2} \partial_{+} \partial_{-} \varphi
$$

while the general solution to $(2.18)$ is

$$
\widehat{\gamma}_{ \pm \pm}=l^{2}\left[\Xi_{ \pm \pm}\left(x^{ \pm}\right)+\partial_{ \pm}^{2} \varphi-\left(\partial_{ \pm} \varphi\right)^{2}\right]
$$

with $\Xi_{ \pm \pm}\left(x^{ \pm}\right) 2$ arbitrary functions of their arguments. Using (2.15), one then gets

$$
A_{ \pm \pm}^{T} \Omega=\widehat{\gamma}_{ \pm \pm}, \quad A_{+-}^{T}=0, \quad \Omega^{2}=16 e^{-4 \varphi} \widehat{\gamma}_{++} \widehat{\gamma}_{--} .
$$

In other words, one can choose $\varphi\left(x^{+}, x^{-}\right), \Xi_{ \pm \pm}\left(x^{ \pm}\right)$as coordinates on solution space and, by expressing (2.15) in terms of these coordinates, we have shown that

The general solution to Einstein's equations with metrics in Fefferman-Graham form is given by

$$
\begin{aligned}
g_{A B} d x^{A} d x^{B} & =\left(-e^{2 \varphi} r^{2}+2 \widehat{\gamma}_{+-}-r^{-2} e^{-2 \varphi}\left(\widehat{\gamma}_{+-}^{2}+\widehat{\gamma}_{++} \widehat{\gamma}_{--}\right)\right) d x^{+} d x^{-}+ \\
& +\widehat{\gamma}_{++}\left(1-r^{-2} e^{-2 \varphi} \widehat{\gamma}_{+-}\right)\left(d x^{+}\right)^{2}+\widehat{\gamma}_{--}\left(1-r^{-2} e^{-2 \varphi} \widehat{\gamma}_{+-}\right)\left(d x^{-}\right)^{2}
\end{aligned}
$$

with $\widehat{\gamma}_{A B}$ defined in equations (2.19) and (2.20).

For instance, in these coordinates, the BTZ black hole[54, 55] is determined by $\varphi=0$ and

$$
\Xi_{ \pm \pm}=2 G\left(M \pm \frac{J}{l}\right)
$$

\subsection{Conformal properties of solution space}

By construction, the finite transformations generated by the spacetime vectors (2.3) leave the Fefferman-Graham form invariant, and furthermore transform solutions to solutions. 
Using light-cone coordinates and the parametrization $\bar{\gamma}_{A B} d x^{A} d x^{B}=-e^{2 \varphi} d x^{+} d x^{-}$, we have

$$
\left\{\begin{array}{l}
\xi^{r}=-\frac{1}{2} \psi r, \quad \psi=\partial_{+} Y^{+}+\partial_{-} Y^{-}+2 \partial_{+} \varphi Y^{+}+2 \partial_{-} \varphi Y^{-}, \\
\xi^{ \pm}=Y^{ \pm}+\frac{l^{2} e^{-2 \varphi}}{2 r^{2}} \partial_{\mp} \psi+O\left(r^{-4}\right),
\end{array}\right.
$$

and get

$$
\begin{aligned}
& \mathcal{L}_{\xi} g_{ \pm \pm} \approx l^{2}\left[Y^{ \pm} \partial_{ \pm} \Xi_{ \pm \pm}+2 \partial_{ \pm} Y^{ \pm} \Xi_{ \pm \pm}-\frac{1}{2} \partial_{ \pm}^{3} Y^{ \pm}\right]+O\left(r^{-2}\right) \\
& \mathcal{L}_{\xi} g_{+-} \approx O\left(r^{-2}\right) .
\end{aligned}
$$

It follows that the local conformal algebra acts on solution space as

$$
-\delta \Xi_{ \pm \pm}=Y^{ \pm} \partial_{ \pm} \Xi_{ \pm \pm}+2 \partial_{ \pm} Y^{ \pm} \Xi_{ \pm \pm}-\frac{1}{2} \partial_{ \pm}^{3} Y^{ \pm}
$$

and with $\delta \varphi=0$. Note that the overall minus sign is convential and choosen so that $\delta \Xi_{ \pm \pm} \equiv \delta_{Y} \Xi_{ \pm \pm}$satisfies $\left[\delta_{Y_{1}}, \delta_{Y_{2}}\right] \Xi_{ \pm \pm}=\delta_{\left[Y_{1}, Y_{2}\right]} \Xi_{ \pm \pm}$.

More generally, when considering the extension of the conformal algebra discussed at the end of section 2.1, we find that

$$
\begin{aligned}
& \mathcal{L}_{\xi} g_{ \pm \pm} \approx l^{2}\left[Y^{ \pm} \partial_{ \pm} \Xi_{ \pm \pm}+2 \partial_{ \pm} Y^{ \pm} \Xi_{ \pm \pm}-\frac{1}{2} \partial_{ \pm}^{3} Y^{ \pm}+\right. \\
&+\left.+\partial_{ \pm}^{2} \omega-2 \partial_{ \pm} \varphi \partial_{ \pm} \omega\right]+O\left(r^{-2}\right), \\
& \mathcal{L}_{\xi} g_{+-} \approx 2 \omega\left(-\frac{r^{2}}{2} e^{2 \varphi}\right)+l^{2} \partial_{+} \partial_{-} \omega+O\left(r^{-2}\right),
\end{aligned}
$$

and thus, that the extended algebra acts on solution space as in (2.24) with in addition $-\delta \varphi=\omega$.

\subsection{Centrally extended surface charge algebra}

Let us take

$$
\varphi=0 .
$$

in this section. In fact, starting from a Fefferman-Graham metric (2.1) with $\bar{\gamma}_{A B}=e^{2 \varphi} \eta_{A B}$ one can obtain such a metric with vanishing $\varphi\left(x^{C}\right)$ through the finite coordinate transformation generated by $\xi^{r}=-\varphi r$ and $\xi^{A}=-l^{2} \partial_{B} \varphi \int_{r}^{\infty} \frac{d r^{\prime}}{r^{\prime}} g^{A B}\left(x, r^{\prime}\right)$ since $\mathcal{L}_{\xi} g_{r r}=0=$ $\mathcal{L}_{\xi} g_{r A}$ and $\mathcal{L}_{\xi} g_{A B}=-2 \varphi g_{A B}$.

The background metric is then

$$
d \bar{s}^{2}=-r^{2} d \tau^{2}+\frac{l^{2}}{r^{2}} d r^{2}+r^{2} d \phi^{2} .
$$


Furthermore,

$$
Y^{+}=Y^{\tau}+Y^{\phi}, Y^{-}=Y^{\tau}-Y^{\phi}, \Lambda=\widehat{\gamma}_{++}+\widehat{\gamma}_{--}, \Sigma=\widehat{\gamma}_{++}-\widehat{\gamma}_{--},
$$

and $g_{A B} d x^{A} d x^{B}=-r^{2} d \tau^{2}+r^{2} d \phi^{2}+h_{A B} d x^{A} d x^{B}$ with

$$
\begin{gathered}
h_{\tau \tau} \approx \Lambda(x)+O\left(r^{-2}\right) \approx h_{\phi \phi}, \quad h_{\tau \phi} \approx \Sigma(x)+O\left(r^{-2}\right), \\
\partial_{\tau} \Lambda=\partial_{\phi} \Sigma, \quad \partial_{\tau} \Sigma=\partial_{\phi} \Lambda .
\end{gathered}
$$

For the surface charges, we follow [56], up to a global change of sign, and use the expression

$$
\begin{aligned}
\mathcal{Q}_{\xi}[g-\bar{g}, \bar{g}]=\int_{\partial \Sigma} \frac{\sqrt{-\bar{g}}}{16 \pi G}\left(d^{n-2} x\right)_{\mu \nu}\left[\xi^{\nu} \bar{D}^{\mu} h-\xi^{\nu} \bar{D}_{\sigma} h^{\mu \sigma}+\xi_{\sigma} \bar{D}^{\nu} h^{\mu \sigma}\right. & \\
& \left.+\frac{1}{2} h \bar{D}^{\nu} \xi^{\mu}+\frac{1}{2} h^{\nu \sigma}\left(\bar{D}^{\mu} \xi_{\sigma}-\bar{D}_{\sigma} \xi^{\mu}\right)-(\mu \leftrightarrow \nu)\right] .
\end{aligned}
$$

Here

$$
\left(d^{n-k} x\right)_{\nu \mu}=\frac{1}{k !(n-k) !} \epsilon_{\nu \mu \alpha_{1} \ldots \alpha_{n-2}} d x^{\alpha_{1}} \wedge \cdots \wedge d x^{\alpha_{n-2}}, \quad \epsilon_{01 \ldots n-1}=1,
$$

with $n=3$ and the surface of integration $\partial \Sigma$ is taken to be the circle at infinity. This gives

$$
\begin{aligned}
& \mathcal{Q}_{\xi}[g-\bar{g}, \bar{g}]=\frac{1}{16 \pi G} \lim _{r \rightarrow \infty} \int_{0}^{2 \pi} r d \phi\left[\xi^{r}\left(\bar{D}^{\tau} h-\bar{D}_{\sigma} h^{\tau \sigma}+\bar{D}^{r} h_{r}^{\tau}-\bar{D}^{\tau} h_{r}^{r}\right)\right. \\
&-\xi^{\tau}\left(\bar{D}^{r} h-\bar{D}_{\sigma} h^{r \sigma}-\bar{D}^{r} h_{\tau}^{\tau}+\bar{D}^{\tau} h_{\tau}^{r}\right)+\xi^{\phi}\left(\bar{D}^{r} h_{\phi}^{\tau}-\bar{D}^{\tau} h_{\phi}^{r}\right)+\frac{1}{2} h\left(\bar{D}^{r} \xi^{\tau}-\bar{D}^{\tau} \xi^{r}\right) \\
&\left.+\frac{1}{2} h^{r \sigma}\left(\bar{D}^{\tau} \xi_{\sigma}-\bar{D}_{\sigma} \xi^{\tau}\right)-\frac{1}{2} h^{\tau \sigma}\left(\bar{D}^{r} \xi_{\sigma}-\bar{D}_{\sigma} \xi^{r}\right)\right] .
\end{aligned}
$$

Using

$$
\begin{gathered}
\bar{D}^{\tau} h-\bar{D}_{\sigma} h^{\tau \sigma}+\bar{D}^{r} h_{r}^{\tau}-\bar{D}^{\tau} h_{r}^{r}=r^{-2} \bar{\gamma}^{A B}\left(\bar{D}_{A} h_{\tau B}-\bar{D}_{\tau} h_{A B}\right)=r^{-4}\left(\partial_{\phi} h_{\tau \phi}-\partial_{\tau} h_{\phi \phi}\right) \\
\bar{D}^{r} h-\bar{D}_{\sigma} h^{r \sigma}-\bar{D}^{r} h_{\tau}^{\tau}+\bar{D}^{\tau} h_{\tau}^{r}=\frac{1}{l^{2}}\left(\partial_{r} h_{\phi \phi}-\frac{1}{r} h_{\tau \tau}\right), \\
\bar{D}^{r} h_{\phi}^{\tau}-\bar{D}^{\tau} h_{\phi}^{r}=-\frac{1}{l^{2}}\left(\partial_{r} h_{\tau \phi}-\frac{1}{r} h_{\tau \phi}\right) \\
\bar{D}^{r} \xi^{\tau}-\bar{D}^{\tau} \xi^{r}=\frac{2 r}{l^{2}} Y^{\tau}-\frac{1}{r} \partial_{\tau} \psi+O\left(r^{-3}\right), \\
\frac{1}{2} h^{r \sigma}\left(\bar{D}^{\tau} \xi_{\sigma}-\bar{D}_{\sigma} \xi^{\tau}\right)-\frac{1}{2} h^{\tau \sigma}\left(\bar{D}^{r} \xi_{\sigma}-\bar{D}_{\sigma} \xi^{r}\right)=\frac{1}{r l^{2}} h_{\tau A} Y^{A}+\frac{1}{4 r} h_{\tau}^{A} \partial_{A} \psi+O\left(r^{-3}\right),
\end{gathered}
$$

we find explicitly

$$
\begin{aligned}
& \mathcal{Q}_{\xi}[g-\bar{g}, \bar{g}]=\frac{1}{16 \pi G l^{2}} \lim _{r \rightarrow \infty} \int_{0}^{2 \pi} d \phi\left(2 Y^{\tau} h_{\phi \phi}+2 Y^{\phi} h_{\tau \phi}\right) \\
& \approx \frac{1}{8 \pi G l^{2}} \int_{0}^{2 \pi} d \phi\left(Y^{\tau} \Lambda+Y^{\phi} \Sigma\right)=\frac{1}{8 \pi G} \int_{0}^{2 \pi} d \phi\left(Y^{+} \Xi_{++}+Y^{-} \Xi_{--}\right) .
\end{aligned}
$$


The considerations of [56] suggest that these charges form a representation of the conformal algebra, or more precisely, that

$$
\begin{gathered}
\mathcal{Q}_{\xi_{1}}\left[\mathcal{L}_{\xi_{2}} g, \bar{g}\right] \approx \mathcal{Q}_{\left[\xi_{1}, \xi_{2}\right]_{M}}[g-\bar{g}, \bar{g}]+K_{\xi_{1}, \xi_{2}}, \\
K_{\xi_{1}, \xi_{2}}=\mathcal{Q}_{\xi_{1}}\left[\mathcal{L}_{\xi_{2}} \bar{g}, \bar{g}\right], \quad\left[\xi_{1}, \xi_{2}\right]_{M}=\left[\xi_{1}, \xi_{2}\right]+\delta_{\xi_{1}}^{g} \xi_{2}-\delta_{\xi_{2}}^{g} \xi_{1} .
\end{gathered}
$$

An asymtotic Killing vector of the form (2.3) depends on the metric, $\xi=\xi[x, g]$ and $\delta_{\xi_{1}}^{g} \xi_{2}=\xi_{2}\left[x, \mathcal{L}_{\xi_{1}} g\right]$. From $\delta_{\xi_{1}}^{g} \xi_{2}^{\tau}=O\left(r^{-4}\right)$ and $\delta_{\xi_{1}}^{g} \xi_{2}^{\phi}=O\left(r^{-4}\right)$, it follows that only the Lie bracket $\left[\xi_{1}, \xi_{2}\right]$ contributes on the right hand side, $\mathcal{Q}_{\left[\xi_{1}, \xi_{2}\right]_{M}}[g-\bar{g}, \bar{g}]=\mathcal{Q}_{\left[\xi_{1}, \xi_{2}\right]}[g-\bar{g}, \bar{g}]$. Using (2.23), 2.27) and integrations by parts in $\partial_{\phi}$ and the conformal Killing equation for $Y_{1}^{A}, Y_{2}^{A}$ to evaluate the left hand side, one indeed finds

$$
\begin{gathered}
\mathcal{Q}_{\xi_{1}}\left[\mathcal{L}_{\xi_{2}} g, \bar{g}\right] \approx \mathcal{Q}_{\left[\xi_{1}, \xi_{2}\right]}[g-\bar{g}, \bar{g}]+K_{\xi_{1}, \xi_{2}}, \\
K_{\xi_{1}, \xi_{2}}=\frac{1}{8 \pi G} \int_{0}^{2 \pi} d \phi\left(\partial_{\phi} Y_{1}^{\tau} \partial_{\phi}^{2} Y_{2}^{\phi}-\partial_{\phi} Y_{2}^{\tau} \partial_{\phi}^{2} Y_{1}^{\phi}\right),
\end{gathered}
$$

where $K_{\xi_{1}, \xi_{2}}$ is a form of the well-known Brown-Henneaux central charge.

In addition, the covariant expression for the surface charges used above coincides onshell with those of the Hamiltonian formalism [56, 57]. In this context, it follows from the analysis of [58, 59, 3] that the surface charge is, after the Fefferman-Graham gauge fixation, the canonical generator of the conformal transformations in the Dirac bracket.

\section{$3 \quad \mathrm{BMS}_{3} / \mathrm{CFT}_{1}$ correspondence}

We consider metrics of the form

$$
d s^{2}=e^{2 \beta} \frac{V}{r} d u^{2}-2 e^{2 \beta} d u d r+r^{2} e^{2 \varphi}(d \phi-U d u)^{2},
$$

or, equivalently,

$$
g_{\mu \nu}=\left(\begin{array}{ccc}
e^{2 \beta} V r^{-1}+r^{2} e^{2 \varphi} U^{2} & -e^{2 \beta} & -r^{2} e^{2 \varphi} U \\
-e^{2 \beta} & 0 & 0 \\
-r^{2} e^{2 \varphi} U & 0 & r^{2} e^{2 \varphi}
\end{array}\right)
$$

with inverse given by

$$
g^{\mu \nu}=\left(\begin{array}{ccc}
0 & -e^{-2 \beta} & 0 \\
-e^{-2 \beta} & -\frac{V}{r} e^{-2 \beta} & -U e^{-2 \beta} \\
0 & -U e^{-2 \beta} & r^{-2} e^{-2 \varphi}
\end{array}\right) .
$$

Here, $\varphi=\varphi(u, \phi)$. Three dimensional Minkowski space is described by $\varphi=0=\beta=U$ and $V=-r$. The fall-off conditions are taken as $\beta=O\left(r^{-1}\right), U=O\left(r^{-2}\right)$ and $V=$ $-2 r^{2} \partial_{u} \varphi+O(r)$. In particular, $g_{u u}=-2 r \partial_{u} \varphi+O(1)$. 


\subsection{Asymptotic symmetries}

The transformations leaving this form of the metric invariant are generated by vector fields such that

$$
\begin{gathered}
\mathcal{L}_{\xi} g_{r r}=0=\mathcal{L}_{\xi} g_{r \phi}, \quad \mathcal{L}_{\xi} g_{\phi \phi}=0 \\
\mathcal{L}_{\xi} g_{u r}=O\left(r^{-1}\right), \quad \mathcal{L}_{\xi} g_{u \phi}=O(1), \quad \mathcal{L}_{\xi} g_{u u}=O(1) .
\end{gathered}
$$

Equations (3.2) imply that

$$
\left\{\begin{array}{l}
\xi^{u}=f \\
\xi^{\phi}=Y+I, \quad I=-e^{-2 \varphi} \partial_{\phi} f \int_{r}^{\infty} d r^{\prime} r^{\prime-2} e^{2 \beta}=-\frac{1}{r} e^{-2 \varphi} \partial_{\phi} f+O\left(r^{-2}\right), \\
\xi^{r}=-r\left[\partial_{\phi} \xi^{\phi}-\partial_{\phi} f U+\xi^{\phi} \partial_{\phi} \varphi+f \partial_{u} \varphi\right],
\end{array}\right.
$$

with $\partial_{r} f=0=\partial_{r} Y$. The first equation of (3.3) then implies that

$$
\partial_{u} f=f \partial_{u} \varphi+Y \partial_{\phi} \varphi+\partial_{\phi} Y \Longleftrightarrow f=e^{\varphi}\left[T+\int_{0}^{u} d u^{\prime} e^{-\varphi}\left(\partial_{\phi} Y+Y \partial_{\phi} \varphi\right)\right],
$$

with $T=T(\phi)$, while the second requires $\partial_{u} Y=0$ and thus $Y=Y(\phi)$, which implies in turn that the last one is identically satisfied.

The Lie algebra $\mathfrak{b m s}_{3}$ is determined by two arbitrary functions $(Y, T)$ on the circle with bracket $\left[\left(Y_{1}, T_{1}\right),\left(Y_{2}, T_{2}\right)\right]=(\widehat{Y}, \widehat{T})$ determined by $\widehat{Y}=Y_{1} \partial_{\phi} Y_{2}-(1 \leftrightarrow 2)$ and $\widehat{T}=Y_{1} \partial_{\phi} T_{2}+T_{1} \partial_{\phi} Y_{2}-(1 \leftrightarrow 2)$. Let $\Im=S^{1} \times \mathbb{R}$ with coordinates $u, \phi$ and consider the vector fields $\bar{\xi}=f \frac{\partial}{\partial u}+Y \frac{\partial}{\partial \phi}$ with $f$ as in (3.5) and $Y=Y(\phi)$. By direct computation, it follows that these vector fields equipped with the commutator bracket provide a faithful representation of $\mathfrak{b m s}_{3}$. Furthermore :

The spacetime vectors (3.4), with $f$ given in (3.5) and $Y=Y(\phi)$ form a faithful representation of the $\mathfrak{b m s}_{3}$ Lie algebra on an asymptotically flat spacetime of the form (3.1) when equipped with the modified bracket $[\cdot, \cdot]_{M}$.

Indeed, for the $u$ component, there is no modification due to the change in the metric and the result follows by direct computation. As a consequence, $\widehat{f}=\left[\xi_{1}, \xi_{2}\right]_{(M)}^{u}$ corresponds to $f$ in (3.5) with $T$ replaced by $\widehat{T}$ and $Y$ by $\widehat{Y}$. By evaluating $\mathcal{L}_{\xi} g^{\mu \nu}$, we find

$$
\left\{\begin{array}{l}
\delta_{\xi} \varphi=0 \\
\delta_{\xi} \beta=\xi^{\alpha} \partial_{\alpha} \beta+\frac{1}{2}\left[\partial_{u} f+\partial_{r} \xi^{r}+\partial_{\phi} f U\right] \\
\delta_{\xi} U=\xi^{\alpha} \partial_{\alpha} U+U\left[\partial_{u} f+\partial_{\phi} f U-\partial_{\phi} \xi^{\phi}\right]-\partial_{u} \xi^{\phi}-\partial_{r} \xi^{\phi} \frac{V}{r}+\partial_{\phi} \xi^{r} \frac{e^{2(\beta-\varphi)}}{r^{2}} .
\end{array}\right.
$$

It follows that

$$
\left\{\begin{array}{l}
\delta_{\xi_{1}}^{g} \xi_{2}^{\phi}=-e^{-2 \varphi} \partial_{\phi} f_{2} \int_{r}^{\infty} \frac{d r^{\prime}}{r^{\prime}} e^{2 \beta} 2 \delta_{\xi_{1}} \beta \\
\delta_{\xi_{1}}^{g} \xi_{2}^{r}=-r\left[\partial_{\phi}\left(\delta_{\xi_{1}}^{g} \xi_{2}^{\phi}\right)+\left(\delta_{\xi_{1}}^{g} \xi_{2}^{\phi}\right) \partial_{\phi} \varphi-\partial_{\phi} f_{2} \delta_{\xi_{1}} U\right]
\end{array}\right.
$$


We also have $\lim _{r \rightarrow \infty}\left[\xi_{1}, \xi_{2}\right]_{M}^{\phi}=\widehat{Y}$. Using $\partial_{r} \xi^{\phi}=\frac{e^{2(\beta-\varphi)}}{r^{2}} \partial_{\phi} f$, (3.5) and the expression of $\xi^{r}$ in (3.4), it follows by a straightforward computation that $\partial_{r}\left(\left[\xi_{1}, \xi_{2}\right]_{M}^{\phi}\right)=\frac{e^{2(\beta-\varphi)}}{r^{2}} \partial_{\phi} \widehat{f}$, which gives the result for the $\phi$ component. Finally, for the $r$ component, we need the relation

$$
\partial_{r}\left(\frac{\xi^{r}}{r}\right)=-\partial_{r}\left(\partial_{\phi} \xi^{\phi}+\xi^{\phi} \partial_{\phi} f \partial_{\phi} \varphi-\partial_{\phi} f U\right) \text {. }
$$

We then have $\lim _{r \rightarrow \infty} \frac{\left[\xi_{1}, \xi_{2}\right]_{M}^{r}}{r}=-\partial_{\phi} \widehat{Y}-\widehat{Y} \partial_{\phi} \varphi-\widehat{f} \partial_{u} \varphi$, while direct computation shows that $\partial_{r}\left(\frac{\left[\xi_{1}, \xi_{2}\right]_{M}^{r}}{r}\right)=-\partial_{r}\left(\partial_{\phi}\left(\left[\xi_{1}, \xi_{2}\right]_{M}^{\phi}\right)-\partial_{\phi}\left(\left[\xi_{1}, \xi_{2}\right]_{M}^{u}\right) U+\left[\xi_{1}, \xi_{2}\right]_{M}^{\phi} \partial_{\phi} \varphi\right)$, which gives the result for the $r$ component.

More generally, one can also consider the transformations that leave the form of the metric (3.1) invariant up to a rescaling of $\varphi$ by $\omega(u, \phi)$. They are generated by spacetime vectors satisfying

$$
\begin{gathered}
\mathcal{L}_{\xi} g_{r r}=0=\mathcal{L}_{\xi} g_{r \phi}, \quad \mathcal{L}_{\xi} g_{\phi \phi}=2 \omega g_{\phi \phi}, \\
\mathcal{L}_{\xi} g_{u r}=O\left(r^{-1}\right), \quad \mathcal{L}_{\xi} g_{u \phi}=O(1), \quad \mathcal{L}_{\xi} g_{u u}=-2 r \partial_{u} \omega+O(1) .
\end{gathered}
$$

Equations (3.8), (3.9) then imply that the vectors are given by (3.4), (3.5) with the replacement $\partial_{\phi} Y \rightarrow \partial_{\phi} Y-\omega$.

With this replacement, the vector fields $\bar{\xi}=f \frac{\partial}{\partial u}+Y \frac{\partial}{\partial \phi}$ on $\Im=S^{1} \times \mathbb{R}$ equipped with the modified bracket provide a faithful representation of the extension of $\mathfrak{b m s}_{3}$ defined by elements $(Y, T, \omega)$ and bracket $\left[\left(Y_{1}, T_{1}, \omega_{1}\right),\left(Y_{2}, T_{2}, \omega_{2}\right)\right]=(\widehat{Y}, \widehat{T}, \widehat{\omega})$, with $\widehat{Y}, \widehat{T}$ as before and $\widehat{\omega}=0$.

Indeed, the result is obvious for the $\phi$ component. Furthermore,

$$
\delta_{\bar{\xi}_{1}}^{g} f_{2}=\omega_{1} f_{2}+e^{\varphi} \int_{0}^{u} d u^{\prime} e^{-\varphi}\left[-\omega_{1}\left(\partial_{\phi} Y_{2}-\omega_{2}+Y_{2} \partial_{\phi} \varphi\right)+Y_{2} \partial_{\phi} \omega_{1}\right]
$$

At $u=0$, we get $\left.\left[\bar{\xi}_{1}, \bar{\xi}_{2}\right]_{M}^{u}\right|_{u=0}=\left.e^{\varphi}\right|_{u=0} \widehat{T}$, while direct computation shows that $\partial_{u}\left(\left[\bar{\xi}_{1}, \bar{\xi}_{2}\right]_{M}^{u}\right)=$ $\widehat{f} \partial_{u} \varphi+\widehat{Y} \partial_{\phi} \varphi+\partial_{\phi} \widehat{Y}$, as it should.

Following the same reasoning as before, one can then also show that the spacetime vectors (3.4) with the replacement discussed above and equipped with the modified Lie bracket provide a faithful representation of the extended $\mathfrak{b m s}_{3}$ algebra.

Indeed, we have $\xi=\bar{\xi}+I \frac{\partial}{\partial \phi}+\xi^{r} \frac{\partial}{\partial r}$. Furthermore, $\left[\xi_{1}, \xi_{2}\right]_{M}^{u}=\left[\bar{\xi}_{1}, \bar{\xi}_{2}\right]_{M}^{u}=\widehat{f}$ as it should. In the extended case, the variations of $\beta, U$ are still given by (3.6). We then have $\lim _{r \rightarrow \infty}\left[\xi_{1}, \xi_{2}\right]_{M}^{\phi}=\widehat{Y}$ and find, after some computations, $\partial_{r}\left(\left[\xi_{1}, \xi_{2}\right]_{M}^{\phi}\right)=\frac{e^{2(\beta-\varphi)}}{r^{2}} \partial_{\phi} \widehat{f}$, giving the result for the $\phi$ component. Finally, we have $\lim _{r \rightarrow \infty} \frac{\left[\xi_{1}, \xi_{2}\right]_{M}^{r}}{r}=-\partial_{\phi} \widehat{Y}-$ $\widehat{Y} \partial_{\phi} \varphi-\widehat{f} \partial_{u} \varphi$, while direct computation shows that $\partial_{r}\left(\frac{\left[\xi_{1}, \xi_{2}\right]_{M}^{r}}{r}\right)=-\partial_{r}\left(\partial_{\phi}\left(\left[\xi_{1}, \xi_{2}\right]_{M}^{\phi}\right)-\right.$ $\left.\partial_{\phi}\left(\left[\xi_{1}, \xi_{2}\right]_{M}^{u}\right) U+\left[\xi_{1}, \xi_{2}\right]_{M}^{\phi} \partial_{\phi} \varphi\right)$, which gives the result for the $r$ component. 


\subsection{Solution space}

Following [52], the equations of motion are organized in terms of the Einstein tensor $G_{\alpha \beta}=R_{\alpha \beta}-\frac{1}{2} g_{\alpha \beta} R$ as

$$
\begin{gathered}
G_{r \alpha}=0, \quad G_{A B}-\frac{1}{2} g_{A B} g^{C D} G_{C D}=0, \\
G_{u u}=0=G_{u A} \\
g^{C D} G_{C D}=0
\end{gathered}
$$

and the Bianchi identities are written as

$$
0=2 \sqrt{-g} G_{\alpha ; \beta}^{\beta}=2\left(\sqrt{-g} G_{\alpha, \beta}^{\beta}\right)_{, \beta} \sqrt{-g} G_{\beta \gamma} g_{, \alpha}^{\beta \gamma} .
$$

For a metric of the form (3.1), we have

$$
\begin{gathered}
\Gamma_{r r}^{\lambda}=\delta_{r}^{\lambda} 2 \beta_{, r}, \quad \Gamma_{\lambda r}^{u}=0, \quad \Gamma_{\phi r}^{r}=\beta_{, \phi}+n, \quad \Gamma_{\phi r}^{\phi}=\frac{1}{r}, \\
\Gamma_{\phi \phi}^{u}=e^{-2 \beta+2 \varphi} r, \quad \Gamma_{\phi \phi}^{\phi}=e^{-2 \beta+2 \varphi} U r+\partial_{\phi} \varphi, \\
\Gamma_{u r}^{\phi}=-\frac{1}{r} U+r^{-2} e^{2 \beta-2 \varphi}\left(\partial_{\phi} \beta-n\right), \quad \Gamma_{u \phi}^{u}=\beta_{, \phi}-n-e^{-2 \beta+2 \varphi} r U, \\
\Gamma_{u r}^{r}=-\frac{1}{2}\left(\partial_{r}+2 \beta_{, r}\right) \frac{V}{r}-\left(\beta_{, \phi}+n\right) U, \quad \Gamma_{\phi u}^{\phi}=\partial_{u} \varphi+U\left(\beta_{, \phi}-n\right)-e^{-2 \beta+2 \varphi} r U^{2}, \\
\Gamma_{u u}^{u}=2 \beta, u+\frac{1}{2}\left(\partial_{r}+2 \beta, r\right) \frac{V}{r}+2 U n+e^{-2 \beta+2 \varphi} r U^{2}, \\
\Gamma_{\phi \phi}^{r}=e^{-2 \beta+2 \varphi}\left(r^{2} \partial_{\phi} U+r^{2} \partial_{\phi} \varphi U+r^{2} \partial_{u} \varphi+V\right), \\
\Gamma_{u \phi}^{r}=-\frac{V, \phi}{2 r}-n \frac{V}{r}-e^{-2 \beta+2 \varphi} U\left[r^{2} \partial_{\phi} U+r^{2} \partial_{\phi} \varphi U-r^{2} \partial_{u} \varphi+V\right], \\
2 U \beta, u+\frac{1}{2} U\left(\partial_{r}+2 \beta{ }_{, r}\right) \frac{V}{r}+2 U^{2} n+r e^{-2 \beta+2 \varphi} U^{3}-U_{, u}-2 \partial_{u} \varphi U \\
-\frac{1}{2} e^{2 \beta-2 \varphi} r^{-2}\left(\partial_{\phi}+2 \partial_{\phi} \beta\right) \frac{V}{r}-U\left(\partial_{\phi}+\partial_{\phi} \varphi\right) U, \\
\Gamma_{u u}^{r}=-\frac{1}{2}\left(\partial_{u}-2 \partial_{u} \beta\right) \frac{V}{r}+\frac{1}{2} \frac{V}{r}\left(\partial_{r}+2 \partial_{r} \beta\right) \frac{V}{r}+V r e^{2 \varphi-2 \beta} U\left(\partial_{r}+\frac{1}{r}\right) U+r^{2} e^{-2 \beta+2 \varphi} U^{2} \partial_{u} \varphi \\
+\frac{1}{2} U\left(\partial_{\phi}+2 \partial_{\phi} \beta\right) \frac{V}{r}+\frac{1}{2} r^{2} e^{-2 \beta+2 \varphi} U\left(\partial_{\phi}+2 \partial_{\phi} \varphi\right) U,
\end{gathered}
$$

where the notation $n=\frac{1}{2} r^{2} e^{2 \varphi-2 \beta} \partial_{r} U$ has been used.

We start with $G_{r r}=0$. From

$$
G_{r r}=R_{r r}=\frac{2}{r} \partial_{r} \beta
$$

we find $\beta=0$ by taking the fall-off conditions into account. From

$$
G_{r \phi}=R_{r \phi}=\left(\partial_{r}+\frac{1}{r}\right) n+\frac{1}{r} \partial_{\phi} \beta,
$$


we then obtain, by using the previous result, that $n=\frac{N}{r}$ where the integration constant $N=N(u, \phi)$. Using the definition of $n$, we get $U=-r^{-2} e^{-2 \varphi} N$. From $G_{r u}=-g^{\phi \phi} R_{\phi \phi}$ and

$$
\begin{aligned}
R_{\phi \phi}=e^{-2 \beta+2 \varphi} & \left(\left(\partial_{r}-\frac{1}{r}\right) V+2 r \partial_{u} \varphi+2 r\left(\partial_{\phi}+\partial_{\phi} \varphi\right) U\right) \\
& -2 \partial_{\phi}^{2} \beta+2 \partial_{\phi} \beta \partial_{\phi} \varphi-2\left(\partial_{\phi} \beta-n\right)^{2}-2 \partial_{\phi} \varphi n+2 \partial_{\phi} n \\
& =e^{2 \varphi}\left(\left(\partial_{r}-\frac{1}{r}\right) V+2 r \partial_{u} \varphi\right)-2 r^{-2} N^{2},
\end{aligned}
$$

we get $\partial_{r}\left(\frac{V}{r}\right)=-2 \partial_{u} \varphi+2 r^{-3} e^{-2 \varphi} N^{2}$ and then

$$
V=-2 r^{2} \partial_{u} \varphi+r M-r^{-1} e^{-2 \varphi} N^{2},
$$

for an additional integration constant $M=M(u, \phi)$.

When $G_{r r}=G_{r \phi}=G_{r u}=0$, the Bianchi identity (3.13) for $\alpha=r$ implies that $G_{\phi \phi}=0$. This implies in turn that $R=0$. The Bianchi identity for $\alpha=\phi$ then gives $\partial_{r}\left(r G_{u \phi}\right)=0$. When $G_{u \phi}=0$, the Bianchi identity for $\alpha=u$ gives $\partial_{r}\left(r G_{u u}\right)=0$. To solve the remaining equations of motion, there thus remain only the constraints

$$
\lim _{r \rightarrow \infty} r R_{u \phi}=0, \quad \quad \lim _{r \rightarrow \infty} r R_{u u}=0 .
$$

to be fulfilled. From

$$
R_{u \phi}=\frac{1}{r}\left(-\left(\partial_{u}+\partial_{u} \varphi\right) N+\frac{1}{2} \partial_{\phi} M\right)+O\left(r^{-2}\right),
$$

we get

$$
N=e^{-\varphi} \Xi(\phi)+e^{-\varphi} \int_{u_{0}}^{u} d \widetilde{u} e^{\varphi} \frac{1}{2} \partial_{\phi} M
$$

while

$$
R_{u u}=\frac{1}{r}\left(-\frac{1}{2}\left(\partial_{u}+2 \partial_{u} \varphi\right) M+e^{-2 \varphi} \partial_{u}\left(\partial_{\phi}^{2} \varphi-\frac{1}{2}\left(\partial_{\phi} \varphi\right)^{2}\right)\right)+O\left(r^{-2}\right)
$$

implies

$$
M=e^{-2 \varphi}\left[\Theta(\phi)-\left(\partial_{\phi} \varphi\right)^{2}+2 \partial_{\phi}^{2} \varphi\right]
$$

We thus have shown:

For metrics of the form (3.1) with $\lim _{r \rightarrow \infty} \beta=0$, the general solution to the equations of motions is given by

$$
\begin{gathered}
d s^{2}=s_{u u} d u^{2}-2 d u d r+2 s_{u \phi} d u d \phi+r^{2} e^{2 \varphi} d \phi^{2} \\
s_{u u}=e^{-2 \varphi}\left[\Theta-\left(\partial_{\phi} \varphi\right)^{2}+2 \partial_{\phi}^{2} \varphi\right]-2 r \partial_{u} \varphi \\
s_{u \phi}=e^{-\varphi}\left[\Xi+\int_{u_{0}}^{u} d \widetilde{u} e^{-\varphi}\left[\frac{1}{2} \partial_{\phi} \Theta-\partial_{\phi} \varphi\left[\Theta-\left(\partial_{\phi} \varphi\right)^{2}+3 \partial_{\phi}^{2} \varphi\right]+\partial_{\phi}^{3} \varphi\right]\right],
\end{gathered}
$$

where $\Theta=\Theta(\phi)$ and $\Xi=\Xi(\phi)$ are arbitrary functions. 


\subsection{Conformal properties of solution space}

By computing $\mathcal{L}_{\xi} s_{\mu \nu}$, we find that the asymptotic symmetry algebra $\mathfrak{b m s}_{3}$ acts on solution space according to

$$
\begin{aligned}
& -\delta \Theta=Y \partial_{\phi} \Theta+2 \partial_{\phi} Y \Theta-2 \partial_{\phi}^{3} Y, \\
& -\delta \Xi=Y \partial_{\phi} \Xi+2 \partial_{\phi} Y \Xi+\frac{1}{2} T \partial_{\phi} \Theta+\partial_{\phi} T \Theta-\partial_{\phi}^{3} T, \\
& -\delta \varphi=0 .
\end{aligned}
$$

For the extended algebra, the first two relations are unchanged, while $-\delta \varphi=\omega$.

\subsection{Centrally extended surface charge algebra}

Let us again take $\varphi=0$ in this section. For the surface charges computed at the circle at infinity $u=c t e, r=$ cte $\rightarrow \infty$, one starts again from (2.28). The background line element, which is used to raise and lower indices, is

$$
d \bar{s}^{2}=-d u^{2}-2 d u d r+r^{2} d \phi^{2}
$$

This gives

$$
\begin{array}{r}
\mathcal{Q}_{\xi}[g-\bar{g}, \bar{g}]=\frac{1}{16 \pi G} \lim _{r \rightarrow \infty} \int_{0}^{2 \pi} r d \phi\left[\xi^{r}\left(\bar{D}^{u} h-\bar{D}_{\sigma} h^{u \sigma}+\bar{D}^{r} h_{r}^{u}-\bar{D}^{u} h_{r}^{r}\right)\right. \\
-\xi^{u}\left(\bar{D}^{r} h-\bar{D}_{\sigma} h^{r \sigma}-\bar{D}^{r} h_{u}^{u}+\bar{D}^{u} h_{u}^{r}\right)+\xi^{\phi}\left(\bar{D}^{r} h_{\phi}^{u}-\bar{D}^{u} h_{\phi}^{r}\right)+\frac{1}{2} h\left(\bar{D}^{r} \xi^{u}-\bar{D}^{u} \xi^{r}\right) \\
\left.+\frac{1}{2} h^{r \sigma}\left(\bar{D}^{u} \xi_{\sigma}-\bar{D}_{\sigma} \xi^{u}\right)-\frac{1}{2} h^{u \sigma}\left(\bar{D}^{r} \xi_{\sigma}-\bar{D}_{\sigma} \xi^{r}\right)\right] .
\end{array}
$$

Using

$$
\begin{gathered}
\bar{D}^{u} h-\bar{D}_{\sigma} h^{u \sigma}+\bar{D}^{r} h_{r}^{u}-\bar{D}^{u} h_{r}^{r}=-\frac{1}{r} h_{u r} \\
\bar{D}^{r} h-\bar{D}_{\sigma} h^{r \sigma}-\bar{D}^{r} h_{u}^{u}+\bar{D}^{u} h_{u}^{r}=-\frac{1}{r} h_{u u}+\frac{2}{r} h_{u r}+\frac{1}{r^{2}} \partial_{\phi} h_{u \phi}, \\
\bar{D}^{r} h_{\phi}^{u}-\bar{D}^{u} h_{\phi}^{r}=\left(\frac{1}{r}-\partial_{r}\right) h_{u \phi}, \\
\bar{D}^{r} \xi^{u}-\bar{D}^{u} \xi^{r}=-2 \partial_{\phi} Y+O(1), \\
\frac{1}{2} h^{r \sigma}\left(\bar{D}^{u} \xi_{\sigma}-\bar{D}_{\sigma} \xi^{u}\right)-\frac{1}{2} h^{u \sigma}\left(\bar{D}^{r} \xi_{\sigma}-\bar{D}_{\sigma} \xi^{r}\right)=-2 \partial_{\phi} Y h_{u r}+\frac{1}{r} h_{u \phi} Y+O\left(r^{-2}\right),
\end{gathered}
$$

we get

$$
\begin{array}{r}
\mathcal{Q}_{\xi}[g-\bar{g}, \bar{g}]=\frac{1}{16 \pi G} \lim _{r \rightarrow \infty} \int_{0}^{2 \pi} d \phi\left[\left(r h_{u r}+u h_{u u}\right) \partial_{\phi} Y+h_{u u} T+2 h_{u \phi} Y\right] \\
\approx \frac{1}{16 \pi G} \int_{0}^{2 \pi} d \phi(\Theta T+2 \Xi Y) .
\end{array}
$$


The surface charges thus provide the inner product that turns the linear spaces of solutions and asymptotic symmetries into dual spaces. It follows that the solutions that we have constructed are all non-trivial as different solutions carry different charges.

From $\delta_{\xi_{1}}^{g} \xi_{2}^{u}=0, \delta_{\xi_{1}}^{g} \xi_{2}^{u}=O\left(r^{-2}\right)$ and $\delta_{\xi_{1}}^{g} \xi_{2}^{r}=O\left(r^{-1}\right)$, it follows that only the Lie bracket $\left[\xi_{1}, \xi_{2}\right]$ contributes on the right hand side of (2.31)- 2.32), $\mathcal{Q}_{\left[\xi_{1}, \xi_{2}\right]_{M}}[g-\bar{g}, \bar{g}]=$ $\mathcal{Q}_{\left[\xi_{1}, \xi_{2}\right]}[g-\bar{g}, \bar{g}]$. Using (3.18), (3.15) and integrations by parts in $\partial_{\phi}$ to evaluate the left hand side, one indeed finds

$$
\begin{aligned}
\mathcal{Q}_{\xi_{1}}\left[\mathcal{L}_{\xi_{2}} g, \bar{g}\right] & \approx \mathcal{Q}_{\left[\xi_{1}, \xi_{2}\right]}[g-\bar{g}, \bar{g}]+K_{\xi_{1}, \xi_{2}}, \\
K_{\xi_{1}, \xi_{2}} & =\frac{1}{8 \pi G} \int_{0}^{2 \pi} d \phi\left[\partial_{\phi} Y_{1}\left(T_{2}+\partial_{\phi}^{2} T_{2}\right)-\partial_{\phi} Y_{2}\left(T_{1}+\partial_{\phi}^{2} T_{1}\right)\right] .
\end{aligned}
$$

where $K_{\xi_{1}, \xi_{2}}$ is the central charget.

\section{$4 \mathrm{BMS}_{4} / \mathrm{CFT}_{2}$ correspondence}

\subsection{Asymptotically flat 4-d spacetimes at null infinity}

Let $x^{0}=u, x^{1}=r, x^{2}=\theta, x^{3}=\phi$ and $A, B, \cdots=2,3$. Following mostly Sachs [7] up to notation, the metric $g_{\mu \nu}$ of an asymptotically flat spacetime can be written in the form

$$
d s^{2}=e^{2 \beta} \frac{V}{r} d u^{2}-2 e^{2 \beta} d u d r+g_{A B}\left(d x^{A}-U^{A} d u\right)\left(d x^{B}-U^{B} d u\right)
$$

where $\beta, V, U^{A}, g_{A B}\left(\operatorname{det} g_{A B}\right)^{-1 / 2}$ are 6 functions of the coordinates, with $\operatorname{det} g_{A B}=$ $r^{4} b(u, \theta, \phi)$ for some fixed function $b(u, \theta, \phi)$. The inverse to the metric

$$
g_{\mu \nu}=\left(\begin{array}{ccc}
e^{2 \beta} \frac{V}{r}+g_{C D} U^{C} U^{D} & -e^{2 \beta} & -g_{B C} U^{C} \\
-e^{2 \beta} & 0 & 0 \\
-g_{A C} U^{C} & 0 & g_{A B}
\end{array}\right)
$$

is given by

$$
g^{\mu \nu}=\left(\begin{array}{ccc}
0 & -e^{-2 \beta} & 0 \\
-e^{-2 \beta} & -\frac{V}{r} e^{-2 \beta} & -U^{B} e^{-2 \beta} \\
0 & -U^{A} e^{-2 \beta} & g^{A B}
\end{array}\right)
$$

The fall-off conditions are

$$
g_{A B} d x^{A} d x^{B}=r^{2} \bar{\gamma}_{A B} d x^{A} d x^{B}+O(r),
$$

Sachs chooses $\bar{\gamma}_{A B}={ }_{0} \gamma_{A B}$ to be the metric on the unit 2 sphere, ${ }_{0} \gamma_{A B} d x^{A} d x^{B}=d \theta^{2}+$ $\sin ^{2} \theta d \phi^{2}$ and $b=\sin ^{2} \theta$, but the geometrical analysis by Penrose [51] suggests to be

\footnotetext{
${ }^{1}$ Due to a change of conventions, some sign errors remain in section 2 of the published version of [9]. See the latest arxiv version for corrections.
} 
somewhat more general and use a metric that is conformal to the latter, for instance, $\bar{\gamma}_{A B} d x^{A} d x^{B}=e^{2 \varphi}\left(d \theta^{2}+\sin ^{2} \theta d \phi^{2}\right)$, with $\varphi=\varphi\left(u, x^{A}\right)$. We will choose the determinant condition more generally to be $b\left(u, x^{A}\right)=\operatorname{det} \bar{\gamma}_{A B}$. In particular, in the above example, on which we now focus, $b=e^{4 \varphi} \sin ^{2} \theta$.

The rest of the fall-off conditions are given by

$$
\beta=O\left(r^{-2}\right), \quad V / r=-2 r \dot{\varphi}-e^{-2 \varphi}+\bar{\Delta} \varphi+O\left(r^{-1}\right), \quad U^{A}=O\left(r^{-2}\right) .
$$

Here, a dot denotes the derivative with respect to $u, \bar{D}_{A}$ denotes the covariant derivative with respect to $\bar{\gamma}_{A B}$. We denote by $\bar{\Gamma}_{B C}^{A}$ the associated Christoffel symbols and by $\bar{\Delta}$ the associated Laplacian. Similiarily, ${ }_{0} D_{A},{ }_{0} \Gamma_{B C}^{A},{ }_{0} \Delta$ correspond to ${ }_{0} \gamma_{A B}$. Note that $g^{A B} g_{B C}=\delta_{C}^{A}$ and that the condition on the determinant implies

$$
\left\{\begin{array}{l}
g^{A B} \partial_{r} g_{A B}=4 r^{-1} \\
g^{A B} \partial_{u} g_{A B}=\bar{\gamma}^{A B} \partial_{u} \bar{\gamma}_{A B}=4 \dot{\varphi} \\
g^{A B} \partial_{C} g_{A B}=\bar{\gamma}^{A B} \partial_{C} \bar{\gamma}_{A B}={ }_{0} \gamma^{A B} \partial_{C 0} \gamma_{A B}+4 \partial_{C} \varphi
\end{array}\right.
$$

where $\bar{\gamma}^{A B} \bar{\gamma}_{B C}=\delta_{C}^{A}={ }_{0} \gamma^{A B}{ }_{0} \gamma_{B C}$. In terms of the metric and its inverse, the fall-off conditions read

$$
\left\{\begin{array}{l}
g_{u u}=-2 r \dot{\varphi}-e^{-2 \varphi}+\bar{\Delta} \varphi+O\left(r^{-1}\right), g_{u r}=-1+O\left(r^{-2}\right), g_{u A}=O(1), \\
g_{r r}=0=g_{r A}, \quad g_{A B}=r^{2} \bar{\gamma}_{A B}+O(r), \\
g^{u r}=-1+O\left(r^{-2}\right), \quad g^{u u}=0=g^{u A} \\
g^{r r}=2 r \dot{\varphi}+e^{-2 \varphi}-\bar{\Delta} \varphi+O\left(r^{-1}\right), g^{r A}=O\left(r^{-2}\right), g^{A B}=r^{-2} \bar{\gamma}^{A B}+O\left(r^{-3}\right) .
\end{array}\right.
$$

With the choice $\varphi=0$, Sachs studies the vector fields that leave invariant this form of the metric with these fall-off conditions. More precisely, he finds the general solution to the equations

$$
\begin{gathered}
\mathcal{L}_{\xi} g_{r r}=0, \quad \mathcal{L}_{\xi} g_{r A}=0, \quad \mathcal{L}_{\xi} g_{A B} g^{A B}=0 \\
\mathcal{L}_{\xi} g_{u r}=O\left(r^{-2}\right), \quad \mathcal{L}_{\xi} g_{u A}=O(1), \quad \mathcal{L}_{\xi} g_{A B}=O(r), \quad \mathcal{L}_{\xi} g_{u u}=O\left(r^{-1}\right) .
\end{gathered}
$$

For arbitrary $\varphi$, the general solution to (4.5) is given by

$$
\left\{\begin{array}{l}
\xi^{u}=f \\
\xi^{A}=Y^{A}+I^{A}, \quad I^{A}=-f_{, B} \int_{r}^{\infty} d r^{\prime}\left(e^{2 \beta} g^{A B}\right) \\
\xi^{r}=-\frac{1}{2} r\left(\psi+\chi-f_{, B} U^{B}+2 f \partial_{u} \varphi\right)
\end{array}\right.
$$

with $\partial_{r} f=0=\partial_{r} Y^{A}$ and where $\psi=\bar{D}_{A} Y^{A}, \chi=\bar{D}_{A} I^{A}$. This gives the expansions

$$
\left\{\begin{array}{l}
\xi^{u}=f, \quad \xi^{A}=Y^{A}-r^{-1} f_{, B} \bar{\gamma}^{B A}+O\left(r^{-2}\right), \\
\xi^{r}=-r\left(f \dot{\varphi}+\frac{1}{2} \psi\right)+\frac{1}{2} \bar{\Delta} f+O\left(r^{-1}\right) .
\end{array}\right.
$$


The first equation of (4.6) then implies that

$$
\dot{f}=f \dot{\varphi}+\frac{1}{2} \psi \Longleftrightarrow f=e^{\varphi}\left[T+\frac{1}{2} \int_{0}^{u} d u^{\prime} e^{-\varphi} \psi\right],
$$

with $T=T(\theta, \phi)$, while the second requires $\partial_{u} Y^{A}=0$ and thus $Y^{A}=Y^{A}(\theta, \phi)$. The third one implies that $Y^{A}$ is a conformal Killing vector of $\bar{\gamma}_{A B}$ and thus also of ${ }_{0} \gamma_{A B}$. The last equation of (4.6) is then satisfied without additional conditions. For the computation, one uses that $\bar{\Delta}=e^{-2 \varphi}{ }_{0} \Delta$ and $\psi={ }_{0} \psi+2 Y^{A} \partial_{A} \varphi$, with ${ }_{0} \psi={ }_{0} D_{A} Y^{A}$ and the following properties of conformal Killing vectors $Y^{A}$ on the unit 2-sphere,

$$
2_{0} D_{B 0} D_{C} Y_{A}={ }_{0} \gamma_{C A 0} D_{B 0} \psi+{ }_{0} \gamma_{A B 0} D_{C 0} \psi-{ }_{0} \gamma_{B C 0} D_{A 0} \psi+2 Y_{C 0} \gamma_{B A}-2 Y_{A 0} \gamma_{B C}
$$

where the indices on $Y^{A}$ are lowered with $\bar{\gamma}_{A B}$. This implies in particular ${ }_{0} \Delta Y^{A}=-Y^{A}$ and also that ${ }_{0} \Delta_{0} \psi=-2_{0} \psi$.

\section{2 $\quad \mathfrak{b m s}_{4}$ Lie algebra}

By definition, the algebra $\mathfrak{b m s}_{4}$ is the semi-direct sum of the Lie algebra of conformal Killing vectors $Y^{A} \frac{\partial}{\partial x^{A}}$ of the unit 2-sphere with the abelian ideal consisting of functions $T\left(x^{A}\right)$ on the 2 -sphere. The bracket is defined through

$$
\begin{aligned}
(\widehat{Y}, \widehat{T}) & =\left[\left(Y_{1}, T_{1}\right),\left(Y_{2}, T_{2}\right)\right], \\
\widehat{Y}^{A} & =Y_{1}^{B} \partial_{B} Y_{2}^{A}-Y_{1}^{B} \partial_{B} Y_{2}^{A}, \\
\widehat{T} & =Y_{1}^{A} \partial_{A} T_{2}-Y_{2}^{A} \partial_{A} T_{1}+\frac{1}{2}\left(T_{10} \psi_{2}-T_{20} \psi_{1}\right) .
\end{aligned}
$$

Let $\Im=\mathbb{R} \times S^{2}$ with coordinates $u, \theta, \phi$. On $\Im$, consider the scalar field $\varphi$ and the vectors fields $\bar{\xi}(\varphi, T, Y)=f \frac{\partial}{\partial u}+Y^{A} \frac{\partial}{\partial x^{A}}$, with $f$ given in (4.9) and $Y^{A}$ an $u$-independent conformal Killing vector of $S^{2}$. It is straightforward to check that these vector fields form a faithful representation of $\mathfrak{b m s}_{4}$ for the standard Lie bracket.

Consider then the modified Lie bracket

$$
\left[\xi_{1}, \xi_{2}\right]_{M}=\left[\xi_{1}, \xi_{2}\right]-\delta_{\xi_{1}}^{g} \xi_{2}+\delta_{\xi_{2}}^{g} \xi_{1}
$$

where $\delta_{\xi_{1}}^{g} \xi_{2}$ denotes the variation in $\xi_{2}$ under the variation of the metric induced by $\xi_{1}$, $\delta_{\xi_{1}}^{g} g_{\mu \nu}=\mathcal{L}_{\xi_{1}} g_{\mu \nu}$,

Spacetime vectors $\xi$ of the form (4.7), with $Y^{A}\left(x^{B}\right)$ a conformal Killing vectors of the 2-sphere and $f\left(u, x^{B}\right)$ satisfying (4.9) provide a faithful representation of $\mathfrak{b m s}_{4}$ when equipped with the modified Lie bracket $[\cdot, \cdot]_{M}$.

Indeed, for the $u$ component, there is no modification due to the change in the metric and the result follows by direct computation: $\left[\xi_{1}, \xi_{2}\right]_{(M)}^{u}=\widehat{f}$, where $\widehat{f}$ corresponds to $f$ 
in (4.9) with $T$ replaced by $\widehat{T}$ and $Y$ by $\widehat{Y}$. By evaluating $\mathcal{L}_{\xi} g^{\mu \nu}$, we find

$$
\left\{\begin{array}{l}
\delta_{\xi} \varphi=0 \\
\delta_{\xi} \beta=\xi^{\alpha} \partial_{\alpha} \beta+\frac{1}{2}\left[\partial_{u} f+\partial_{r} \xi^{r}+\partial_{A} f U^{A}\right], \\
\delta_{\xi} U^{A}=\xi^{\alpha} \partial_{\alpha} U^{A}+U^{A}\left(\partial_{u} f+\partial_{B} f U^{B}\right)-\partial_{B} \xi^{A} U^{B} \\
\quad-\partial_{u} \xi^{A}-\partial_{r} \xi^{A} \frac{V}{r}+\partial_{B} \xi^{r} g^{A B} e^{2 \beta} .
\end{array}\right.
$$

It follows that

$$
\left\{\begin{array}{l}
\delta_{\xi_{1}}^{g} \xi_{2}^{A}=-\partial_{B} f_{2} \int_{r}^{\infty} d r^{\prime} e^{2 \beta}\left(2 \delta_{\xi_{1}} \beta g^{A B}+\mathcal{L}_{\xi} g^{A B}\right) \\
\delta_{\xi_{1}}^{g} \xi_{2}^{r}=-\frac{1}{2} r\left[\bar{D}_{A}\left(\delta_{\xi_{1}}^{g} \xi_{2}^{A}\right)-\partial_{A} f_{2} \delta_{\xi_{1}} U^{A}\right]
\end{array}\right.
$$

We have $\lim _{r \rightarrow \infty}\left[\xi_{1}, \xi_{2}\right]_{M}^{A}=\widehat{Y}^{A}$ and, using $\partial_{r} \xi^{A}=g^{A B} e^{2 \beta} \partial_{B} f$, (4.9) together with the expression of $\xi^{r}$ in (4.8), it follows by a straightforward computation that $\partial_{r}\left(\left[\xi_{1}, \xi_{2}\right]_{M}^{A}\right)=$ $g^{A B} e^{2 \beta} \partial_{B} \widehat{f}$, which gives the result for the $A$ components. Finally, for the $r$ component, we need

$$
\partial_{r}\left(\frac{\xi^{r}}{r}\right)=-\frac{1}{2}\left(\partial_{r} \chi-\partial_{B} f \partial_{r} U\right)
$$

We then find $\lim _{r \rightarrow \infty} \frac{\left[\xi_{1}, \xi_{2}\right]_{M}^{r}}{r}=-\frac{1}{2}\left(\widehat{\psi}+2 \widehat{f} \partial_{u} \varphi\right)$, where $\widehat{\psi}$ corresponds to $\psi$ with $Y^{A}$ replaced by $\widehat{Y}^{A}$, while $\partial_{r}\left(\frac{\left[\xi_{1}, \xi_{2}\right]_{M}^{r}}{r}\right)=-\frac{1}{2}\left(\partial_{r} \widehat{\chi}-\partial_{B} \widehat{f} \partial_{r} U^{B}\right)$, where $\widehat{\chi}$ corresponds to $\chi$ with $f$ replaced by $\widehat{f}$. This gives the result for the $r$ component and concludes the proof.

In terms of the standard complex coordinates $\zeta=e^{i \phi} \cot \frac{\theta}{2}$, the metric on the sphere is conformally flat,

$$
d \theta^{2}+\sin ^{2} \theta d \phi^{2}=P^{-2} d \zeta d \bar{\zeta}, \quad P(\zeta, \bar{\zeta})=\frac{1}{2}(1+\zeta \bar{\zeta})
$$

and, since conformal Killing vectors are invariant under conformal rescalings of the metric, the conformal Killing vectors of the unit sphere are the same as the conformal Killing vectors of the Riemann sphere.

Depending on the space of functions under consideration, there are then basically two options which define what is actually meant by $\mathfrak{b m s}_{4}$.

The first choice consists in restricting oneself to globally well-defined transformations on the unit or, equivalently, the Riemann sphere. This singles out the global conformal transformations, also called projective transformations, and the associated group is isomorphic to $S L(2, \mathbb{C}) / \mathbb{Z}_{2}$, which is itself isomorphic to the proper, orthochronous Lorentz group. Associated with this choice, the functions $T(\theta, \phi)$, which are the generators of the so-called supertranslations, have been expanded into spherical harmonics. This choice has been adopted in the original work by Bondi, van der Burg, Metzner and Sachs and followed ever since, most notably in the work of Penrose and Newman-Penrose [ [51, 60], where spin-weighted spherical harmonics and the associated "edth" operator have made their appearance. After attempts to cut this group down to the standard Poincare group, it has been taken seriously as an invariance group of asymptotically flat spacetimes. Its 
consequences have been investigated, but we believe that it is fair to say that this version of the BMS group has had only a limited amount of success.

The second choice that we would like to advocate here is motivated by exactly the same considerations that are at the origin of the breakthrough in two dimensional conformal quantum field theories [61]. It consists in focusing on local properties and allowing the set of all, not necessarily invertible holomorphic mappings. In this case, Laurent series on the Riemann sphere are allowed. The general solution to the conformal Killing equations is $Y^{\zeta}=Y(\zeta), Y^{\bar{\zeta}}=\bar{Y}(\bar{\zeta})$, with $Y$ and $\bar{Y}$ independent functions of their arguments. The standard basis vectors are choosen as

$$
l_{n}=-\zeta^{n+1} \frac{\partial}{\partial \zeta}, \quad \bar{l}_{n}=-\bar{\zeta}^{n+1} \frac{\partial}{\partial \bar{\zeta}}, \quad n \in \mathbb{Z}
$$

At the same time, let us choose to expand the generators of the supertranslations in terms of

$$
T_{m, n}=P^{-1} \zeta^{m} \bar{\zeta}^{n}, \quad m, n \in \mathbb{Z} .
$$

In terms of the basis vector $l_{l} \equiv\left(l_{l}, 0\right)$ and $T_{m n}=\left(0, T_{m n}\right)$, the commutation relations for the complexified $\mathfrak{b m s}_{4}$ algebra read

$$
\begin{aligned}
& {\left[l_{m}, l_{n}\right]=(m-n) l_{m+n}, \quad\left[\bar{l}_{m}, \bar{l}_{n}\right]=(m-n) \bar{l}_{m+n}, \quad\left[l_{m}, \bar{l}_{n}\right]=0} \\
& {\left[l_{l}, T_{m, n}\right]=\left(\frac{l+1}{2}-m\right) T_{m+l, n}, \quad\left[\bar{l}_{l}, T_{m, n}\right]=\left(\frac{l+1}{2}-n\right) T_{m, n+l} .}
\end{aligned}
$$

The $\mathfrak{b m s}_{4}$ algebra contains as subalgebra the Poincaré algebra, which we identify with the algebra of exact Killing vectors of the Minkowski metric equipped with the standard Lie bracket.

Indeed, these vectors form the subspace of spacetime vectors 4.7) for which (i) $\beta=$ $0=U^{A}=\varphi$ while $V=-r$ and $g_{A B}={ }_{0} \gamma_{A B}$ and (ii) the relations in (4.6) hold with 0 on the right hand sides. The former implies in particular that $I^{A}=-\frac{1}{r} 0 \gamma^{A B} \partial_{B} f$, while a first consequence of the latter is that the modified Lie bracket reduces the standard one.

Besides the previous conditions that $Y^{A}$ is an $u$-independent conformal Killing vector of the 2-sphere, $\mathcal{L}_{Y 0} \gamma_{A B}={ }_{0} D_{C} Y^{C}{ }_{0} \gamma_{A B}$ and $f=T+\frac{1}{2} u_{0} \psi$ with $T_{, u}=0=T_{, r}$, we find the additional constraints

$$
\begin{gathered}
{ }_{0} D_{A} \partial_{B}{ }_{0} \psi+{ }_{0} D_{B} \partial_{A 0} \psi={ }_{0} \gamma_{A B}{ }_{0} \Delta_{0} \psi, \\
{ }_{0} D_{A} \partial_{B} T+{ }_{0} D_{B} \partial_{A} T={ }_{0} \gamma_{A B}{ }_{0} \Delta T, \quad \partial_{A} T=-\frac{1}{2} \partial_{A}\left({ }_{0} \Delta T\right) .
\end{gathered}
$$

In the coordinates $\zeta, \bar{\zeta}$, these constraints are equivalent to $\partial^{3} Y=0=\bar{\partial}^{3} \bar{Y}$ and $\partial^{2} \widetilde{T}=$ $0=\bar{\partial}^{2} \widetilde{T}$, where $T=P^{-1} \widetilde{T}$ and $\partial=\frac{\partial}{\partial \zeta}, \bar{\partial}=\frac{\partial}{\partial \bar{\zeta}}$, so that the complexified Poincaré algebra is spanned by the generators

$$
l_{-1}, l_{0}, l_{1}, \quad \bar{l}_{-1}, \bar{l}_{0}, \bar{l}_{1}, \quad T_{0,0}, T_{1,0}, T_{0,1}, T_{1,1},
$$


and the non vanishing commutation relations read

$$
\begin{gathered}
{\left[l_{-1}, l_{0}\right]=-l_{-1},\left[l_{-1}, l_{1}\right]=-2 l_{0},\left[l_{0}, l_{1}\right]=-l_{1},} \\
{\left[l_{-1}, T_{1,0}\right]=-T_{0,0},\left[l_{-1}, T_{1,1}\right]=-T_{0,1},\left[\bar{l}_{-1}, T_{0,1}\right]=-T_{0,0},\left[\bar{l}_{-1}, T_{1,1}\right]=-T_{1,0}} \\
{\left[l_{0}, T_{0,0}\right]=\frac{1}{2} T_{0,0},\left[l_{0}, T_{0,1}\right]=\frac{1}{2} T_{0,1},\left[l_{0}, T_{1,0}\right]=-\frac{1}{2} T_{1,0},\left[l_{0}, T_{1,1}\right]=-\frac{1}{2} T_{1,1}} \\
{\left[\bar{l}_{0}, T_{0,0}\right]=\frac{1}{2} T_{0,0},\left[\bar{l}_{0}, T_{0,1}\right]=-\frac{1}{2} T_{0,1},\left[\bar{l}_{0}, T_{1,0}\right]=\frac{1}{2} T_{1,0},\left[\bar{l}_{0}, T_{1,1}\right]=-\frac{1}{2} T_{1,1}} \\
{\left[l_{1}, T_{0,0}\right]=T_{1,0},\left[l_{1}, T_{0,1}\right]=T_{1,1},\left[\bar{l}_{1}, T_{0,0}\right]=T_{0,1},\left[\bar{l}_{1}, T_{1,0}\right]=T_{1,1} .}
\end{gathered}
$$

In particular for instance, the generators for translations can be written as $\frac{1}{2}\left(T_{1,1}+T_{0,0}\right)=$ $1, \frac{1}{2}\left(T_{1,1}-T_{0,0}\right)=\cos \theta, \frac{1}{2}\left(T_{1,0}+T_{0,1}\right)=\sin \theta \cos \phi, \frac{1}{2 i}\left(T_{1,0}-T_{0,1}\right)=\sin \theta \sin \phi$. Note that in order for the asymptotic symmetry algebra to contain the Poincaré algebra as a subalgebra, it is essential not to restrict the generators of supertranslations to the sum of holomorphic and antiholomorphic functions on the Riemann sphere.

The considerations above apply for all choices of $\varphi$ which is freely at our disposal. In the original work of Bondi, van der Burg, Metzner and Sachs, and in much of the subsequent work, the choice $\varphi=0$ was preferred. From the conformal point of view, the choice

$$
\varphi=\ln \left[\frac{1}{2}(1+\zeta \bar{\zeta})\right]
$$

is interesting as it turns $\bar{\gamma}_{A B}$ into the flat metric on the Riemann sphere with vanishing Christoffel symbols,

$$
\bar{\gamma}_{A B} d x^{A} d x^{B}=d \zeta d \bar{\zeta}
$$

In this case, $\psi=\partial_{A} Y^{A}$,

$$
f=\widetilde{T}+\frac{1}{2} u \psi
$$

with $\widetilde{T}=P T$. In terms of $\widetilde{T}$, we get instead of the last of 4.11

$$
\widehat{\widetilde{T}}=Y_{1}^{A} \widetilde{T}_{2}+\frac{1}{2} \widetilde{T}_{1} \partial_{A} Y_{2}^{A}-(1 \leftrightarrow 2) .
$$

In terms of generators, the algebra (4.17) is unchanged if one now expands the supertranslations $\widetilde{T}$ directly in terms of $\widetilde{T}_{m, n}=\zeta^{m} \bar{\zeta}^{n}$.

More generally, one can also consider the transformations that leave the form of the metric (4.1) invariant up to a conformal rescaling of $g_{A B}$, i.e., up to a rescaling of $\varphi$ by $\omega\left(u, x^{A}\right)$. They are generated by spacetime vectors satisfying

$$
\begin{aligned}
& \mathcal{L}_{\xi} g_{r r}=0, \quad \mathcal{L}_{\xi} g_{r A}=0, \quad \mathcal{L}_{\xi} g_{A B} g^{A B}=4 \omega \\
\mathcal{L}_{\xi} g_{u r}= & O\left(r^{-2}\right), \quad \mathcal{L}_{\xi} g_{u A}=O(1), \quad \mathcal{L}_{\xi} g_{A B}=2 \omega g_{A B}+O(r), \\
& \mathcal{L}_{\xi} g_{u u}=-2 r \dot{\omega}-2 \omega e^{-2 \varphi}+2 \omega \bar{\Delta} \varphi+O\left(r^{-1}\right) .
\end{aligned}
$$


Equations (4.26), (4.27) then imply that the vectors are given by (4.7) and (4.9) with the replacement $\psi \rightarrow \psi-2 \omega$.

With this replacement, the vector fields $\bar{\xi}=f \frac{\partial}{\partial u}+Y^{A} \frac{\partial}{\partial x^{A}}$ on $\Im=\mathbb{R} \times S^{2}$ equipped with the modified bracket provide a faithful representation of the extension of $\mathfrak{b m s}_{4}$ defined by elements $(Y, T, \omega)$ and bracket $\left[\left(Y_{1}, T_{1}, \omega_{1}\right),\left(Y_{2}, T_{2}, \omega_{2}\right)\right]=(\widehat{Y}, \widehat{T}, \widehat{\omega})$, with $\widehat{Y}, \widehat{T}$ as before and $\widehat{\omega}=0$.

Indeed, the result is obvious for the $A$ components. Furthermore,

$$
\delta_{\bar{\xi}_{1}}^{g} f_{2}=\omega_{1} f_{2}+\frac{1}{2} e^{\varphi} \int_{0}^{u} d u^{\prime} e^{-\varphi}\left[-\omega_{1}\left(\psi_{2}-2 \omega_{2}\right)+2 Y_{2}^{A} \partial_{A} \omega_{1}\right] .
$$

At $u=0$, we get $\left.\left[\bar{\xi}_{1}, \bar{\xi}_{2}\right]_{M}^{u}\right|_{u=0}=\left.e^{\varphi}\right|_{u=0} \widehat{T}$, while direct computation shows that $\partial_{u}\left(\left[\bar{\xi}_{1}, \bar{\xi}_{2}\right]_{M}^{u}\right)=$ $\widehat{f} \dot{\varphi}+\frac{1}{2} \bar{D}_{B} \widehat{Y}^{B}$, as it should.

Following the same reasoning as before, one can then also show that the spacetime vectors (4.7) with the replacement discussed above and equipped with the modified Lie bracket provide a faithful representation of the extended $\mathfrak{b m s}_{4}$ algebra.

Indeed, we have $\xi=\bar{\xi}+I^{A} \frac{\partial}{\partial x^{A}}+\xi^{r} \frac{\partial}{\partial r}$. Furthermore, $\left[\xi_{1}, \xi_{2}\right]_{M}^{u}=\left[\bar{\xi}_{1}, \bar{\xi}_{2}\right]_{M}^{u}=\widehat{f}$ as it should. In the extended case, the variations of $\beta, U^{A}$ are still given by (4.13). We then have $\lim _{r \rightarrow \infty}\left[\xi_{1}, \xi_{2}\right]_{M}^{A}=\widehat{Y}^{A}$ and find, after some computations, $\partial_{r}\left(\left[\xi_{1}, \xi_{2}\right]_{M}^{A}\right)=$ $g^{A B} e^{2 \beta} \partial_{B} \widehat{f}$, giving the result for the $A$ components. Finally, for the $r$ component, we find $\lim _{r \rightarrow \infty} \frac{\left[\xi_{1}, \xi_{2}\right]_{M}^{r}}{r}=-\frac{1}{2}(\widehat{\psi}+2 \widehat{f} \dot{\varphi})$, while $\partial_{r}\left(\frac{\left[\xi_{1}, \xi_{2}\right]_{M}^{r}}{r}\right)=-\frac{1}{2}\left(\partial_{r} \widehat{\chi}-\partial_{B} \widehat{f} \partial_{r} U^{B}\right)$, which concludes the proof.

In order to make contact with the original literature, we have choosen the conformal factor with respect to the unit sphere, $\bar{\gamma}_{A B}=e^{2 \varphi}{ }_{0} \gamma_{A B}$. Computations could have been simplified and the derivation of algebra (4.17) would have been streamlined by introducing the conformal factor directly with respect to the flat metric, $\bar{\gamma}_{A B} d x^{A} d x^{B}=e^{2 \widetilde{\varphi}} d \zeta d \bar{\zeta}$, with $\widetilde{\varphi}(u, \zeta, \bar{\zeta})=\varphi-\ln P$, as presented in [8]. In this case, the determinant condition is taken as $b=\frac{1}{4} e^{4 \widetilde{\varphi}}$, while the boundary condition that involves the conformal factor now reads

$$
\frac{V}{r}=-2 r \partial_{u} \widetilde{\varphi}+\bar{\Delta} \widetilde{\varphi}+O\left(r^{-1}\right)
$$

where $\bar{\Delta} \widetilde{\varphi}=4 e^{-2 \widetilde{\varphi}} \partial \bar{\partial} \widetilde{\varphi}$ with $\partial=\partial_{\zeta}, \bar{\partial}=\partial_{\bar{\zeta}}$.

\subsection{Solution space}

We start by assuming only that we have a metric of the form (4.1) and that the determinant condition holds. Following again [52], the equations of motion are organized in terms of 
the Einstein tensor $G_{\alpha \beta}=R_{\alpha \beta}-\frac{1}{2} g_{\alpha \beta} R$ as

$$
\begin{gathered}
G_{r \alpha}=0, \quad G_{A B}-\frac{1}{2} g_{A B} g^{C D} G_{C D}=0, \\
G_{u u}=0=G_{u A} \\
g^{C D} G_{C D}=0 .
\end{gathered}
$$

Due to the form of the metric and the determinant condition, equation (4.31) is a consequence of (4.29) on account of the Bianchi identities. Indeed, the latter can be written as

$$
0=2 \sqrt{-g} G_{\alpha ; \beta}^{\beta}=2\left(\sqrt{-g} G_{\alpha}^{\beta}\right)_{, \beta}+\sqrt{-g} G_{\beta \gamma} g_{, \alpha}^{\beta \gamma} .
$$

When (4.29) hold and $\alpha=1$, we get $G_{A B} g^{A B}{ }_{, r}=0=\frac{1}{2} g_{A B} g^{A B}{ }_{, r} g^{C D} G_{C D}$. This implies that (4.31) holds by using (4.4).

The remaining Bianchi identities then reduce to $2\left(\sqrt{-g} G_{A}^{\beta}\right)_{, \beta}=0=2\left(\sqrt{-g} G_{u}^{\beta}\right)_{, \beta}$. The first gives $\left(r^{2} G_{u A}\right)_{, r}=0$. This means that if $r^{2} G_{u A}=0$ for some constant $r$, it vanishes for all $r$. When $G_{u A}=0$, the last Bianchi identity reduces to $\left(r^{2} G_{u u}\right)_{, r}=0$, so that again, $r^{2} G_{u u}=0$ everywhere if it vanishes for some fixed $r$.

Let $k_{A B}=\frac{1}{2} g_{A B, r}, l_{A B}=\frac{1}{2} g_{A B, u}, n_{A}=\frac{1}{2} e^{-2 \beta} g_{A B} U_{, r}^{B}$ with indices on these variables and on $U^{A}$ lowered and raised with the 2 dimensional metric $g_{A B}$ and its inverse. Define $K_{B}^{A}$ through the relation $k_{B}^{A}=\frac{1}{r} \delta_{B}^{A}+\frac{1}{r^{2}} K_{B}^{A}$. In particular, the determinant condition implies that $k=\frac{2}{r}$ and thus that $K_{D}^{D}=0$. Similarily, if $l_{B}^{D}=\frac{1}{2} \bar{\gamma}^{D A} \bar{\gamma}_{A B, u}+\frac{1}{r} L_{B}^{D}$, the determinant condition implies in particular that $L_{B}^{D}$ is traceless, $L_{D}^{D}=0$. Note that for a traceless $2 \times 2$ matrix $M_{B}^{T}$, we have $M_{C}^{T}{ }_{C}^{A} M_{B}^{T}{ }_{B}^{C}=\frac{1}{2} M^{T}{ }_{D}^{C} M^{T}{ }_{C}^{D} \delta_{B}^{A}$. 
For a metric of the form (4.1), we have

$$
\begin{aligned}
& \Gamma_{r r}^{\lambda}=\delta_{r}^{\lambda} 2 \beta_{, r}, \quad \Gamma_{\lambda r}^{u}=0, \quad \Gamma_{A r}^{r}=\beta_{, A}+n_{A}, \quad \Gamma_{B r}^{A}=k_{B}^{A}, \\
& \Gamma_{A B}^{u}=e^{-2 \beta} k_{A B}, \quad \Gamma_{B C}^{A}=e^{-2 \beta} U^{A} k_{B C}+{ }^{(2)} \Gamma_{B C}^{A}, \\
& \Gamma_{u r}^{A}=-k_{B}^{A} U^{B}+e^{2 \beta}\left(\partial^{A} \beta-n^{A}\right), \quad \Gamma_{u A}^{u}=\beta_{, A}-n_{A}-e^{-2 \beta} k_{A B} U^{B}, \\
& \Gamma_{u r}^{r}=-\frac{1}{2}\left(\partial_{r}+2 \beta_{, r}\right) \frac{V}{r}-\left(\beta_{, A}+n_{A}\right) U^{A}, \\
& \Gamma_{B u}^{A}=l_{B}^{A}+\frac{1}{2}{ }^{(2)} D^{A} U_{B}-\frac{1}{2}{ }^{(2)} D_{B} U^{A}+U^{A}\left(\beta_{, B}-n_{B}\right)-e^{-2 \beta} k_{B C} U^{A} U^{C}, \\
& \Gamma_{u u}^{u}=2 \beta_{, u}+\frac{1}{2}\left(\partial_{r}+2 \beta_{, r}\right) \frac{V}{r}+2 U^{A} n_{A}+e^{-2 \beta} k_{A B} U^{A} U^{B}, \\
& \Gamma_{A B}^{r}=e^{-2 \beta}\left(\frac{1}{2}{ }^{(2)} D_{A} U_{B}+\frac{1}{2}{ }^{(2)} D_{B} U_{A}+l_{A B}+k_{A B} \frac{V}{r}\right), \\
& \Gamma_{u A}^{r}=-\left(\frac{V, A}{2 r}+\frac{V}{r} n_{A}+e^{-2 \beta} U^{B}\left[\frac{1}{2}{ }^{(2)} D_{A} U_{B}+\frac{1}{2}{ }^{(2)} D_{B} U_{A}+l_{A B}+\frac{V}{r} k_{A B}\right]\right) \text {, } \\
& \Gamma_{u u}^{A}=2 U^{A} \beta_{, u}+\frac{1}{2} U^{A}\left(\partial_{r}+2 \beta_{, r}\right) \frac{V}{r}+2 U^{A} n_{B} U^{B}+U^{A} k_{B C} e^{-2 \beta} U^{B} U^{C} \\
& -U_{, u}^{A}-2 l_{B}^{A} U^{B}-\frac{1}{2} e^{2 \beta}\left(\partial^{A}+2 \partial^{A} \beta\right) \frac{V}{r}-\frac{1}{2}{ }^{(2)} D^{A}\left(U^{C} U_{C}\right), \\
& \Gamma_{u u}^{r}=-\frac{1}{2}\left(\partial_{u}-2 \beta_{, u}\right) \frac{V}{r}+\frac{1}{2} \frac{V}{r}\left(\partial_{r}+2 \beta_{, r}\right) \frac{V}{r}+\frac{1}{2} U^{A}\left(\partial_{A}+2 \beta_{, A}\right) \frac{V}{r}+2 \frac{V}{r} U^{A} n_{A} \\
& +\frac{V}{r} e^{-2 \beta} k_{A B} U^{A} U^{B}+e^{-2 \beta} l_{A B} U^{A} U^{B}+e^{-2 \beta} U^{A} U^{B(2)} D_{A} U_{B} .
\end{aligned}
$$

To write the equations of motion, we use that $\left|{ }^{(4)} g\right|=\left.e^{4 \beta}\right|^{(2)} g \mid$ and

$$
R_{\mu \nu}=\left[\partial_{\alpha}+\left(2 \beta+\frac{1}{2} \ln \left|{ }^{(2)} g\right|\right)_{, \alpha}\right] \Gamma_{\mu \nu}^{\alpha}-\partial_{\mu} \partial_{\nu}\left(2 \beta+\frac{1}{2} \ln \left|{ }^{(2)} g\right|\right)-\Gamma_{\nu \beta}^{\alpha} \Gamma_{\mu \alpha}^{\beta} .
$$

The equation $G_{r r} \equiv R_{r r}=0$ then becomes

$$
\partial_{r} \beta=-\frac{1}{2 r}+\frac{r}{4} k_{B}^{A} k_{A}^{B}=\frac{1}{4 r^{3}} K_{B}^{A} K_{A}^{B} \Longleftrightarrow \beta=-\int_{r}^{\infty} d r^{\prime} \frac{1}{4 r^{\prime 3}} K_{B}^{A} K_{A}^{B} .
$$

This equation determines $\beta$ uniquely in terms of $g_{A B}$ because the fall-off condition (4.3) excludes the arbitrary function of $u, x^{A}$ allowed by the general solution to this equation.

The equations $G_{r A} \equiv R_{r A}=0$ read

$$
\begin{gathered}
\partial_{r}\left(r^{2} n_{A}\right)=J_{A} \\
J_{A}=r^{2}\left(\partial_{r}-\frac{2}{r}\right) \beta_{, A}-{ }^{(2)} D_{B} K_{A}^{B}=\partial_{A}\left(-2 r \beta+\frac{1}{4 r} K_{C}^{B} K_{B}^{C}\right)-{ }^{(2)} D_{B} K_{A}^{B} .
\end{gathered}
$$

In the original approach [5, 6], it was assumed in particular that the metric $g_{A B}$ admits an expansion in terms of powers of $r^{-1}$ starting at order $r^{2}$. We will assume

$$
g_{A B}=r^{2} \bar{\gamma}_{A B}+r C_{A B}+D_{A B}+\frac{1}{4} \bar{\gamma}_{A B} C_{D}^{C} C_{C}^{D}+o\left(r^{-\epsilon}\right)
$$


where indices on $C_{A B}, D_{A B}$ are raised with the inverse of $\bar{\gamma}_{A B}$. In [52], it was then shown explicitly how (4.35) is related to the conformal approach [51, 62] and imposed through differentiability conditions at null infinity.

Under the assumption (4.35), $C_{D}^{D}=0=D_{C}^{C}$ and

$$
\begin{aligned}
K_{B}^{A} & =-\frac{1}{2} C_{B}^{A}-r^{-1} D_{B}^{A}+o\left(r^{-1-\epsilon}\right), \\
\beta & =-\frac{1}{32} r^{-2} C_{B}^{A} C_{A}^{B}-\frac{1}{12} r^{-3} C_{B}^{A} D_{A}^{B}+o\left(r^{-3-\epsilon}\right), \\
J_{A} & =\frac{1}{2} \bar{D}_{B} C_{A}^{B}+r^{-1} \bar{D}_{B} D_{A}^{B}+o\left(r^{-1-\epsilon}\right) .
\end{aligned}
$$

These equations then imply $n_{A}=\frac{1}{2} r^{-1} \bar{D}_{B} C_{A}^{B}+r^{-2}\left(\ln r \bar{D}_{B} D_{A}^{B}+N_{A}\right)+o\left(r^{-2-\epsilon}\right)$ and involve the arbitrary functions $N_{A}\left(u, x^{B}\right)$ as integration "constants". Because $U^{A}$ has to vanish for $r \rightarrow \infty$, we get from the definition of $n_{A}$

$$
U^{A}=-\frac{1}{2} r^{-2} \bar{D}_{B} C^{B A}-\frac{2}{3} r^{-3}\left[\left(\ln r+\frac{1}{3}\right) \bar{D}_{B} D^{B A}-\frac{1}{2} C_{B}^{A} \bar{D}_{C} C^{C B}+N^{A}\right]+o\left(r^{-3-\varepsilon}\right),
$$

where the index on $N_{A}$ has been raised with $\bar{\gamma}^{A B}$.

It is straightforward to verify that if one trades the coordinate $r$ for $s=r^{-1}$, the only non vanishing components of the "unphysical" Weyl tensor at the boundary are given by

$$
\lim _{s \rightarrow 0}\left(s^{2} W_{s A s B}\right)=-D_{A B}
$$

(see e.g. [53] for a detailed discussion). In [6], the condition $D_{A B}=0$ was imposed in order to avoid a logarithmic $r$-dependence in the solution to the equations of motion and to avoid singularities on the unit sphere. When one dispenses with this latter restriction, absence of a logarithmic $r$-dependence is guaranteed through the requirement $\bar{D}_{B} D^{B A}=$ 0 . In the coordinates $\zeta, \bar{\zeta}$ and with the parametrization $\bar{\gamma}_{A B} d x^{A} d x^{B}=e^{2 \widetilde{\varphi}} d \zeta d \bar{\zeta}$, this is equivalent to

$$
D_{\zeta \zeta}=d(u, \zeta), \quad D_{\bar{\zeta} \bar{\zeta}}=\bar{d}(u, \bar{\zeta}), \quad D_{\zeta \bar{\zeta}}=0 .
$$

A more complete analysis of the field equations when allowing for a logarithmic or, more precisely, a "polyhomogeneous" dependence in $r$ can be found in [63].

Starting from

$$
\begin{aligned}
R_{A B}=\left(\partial_{r}+2 \beta_{, r}+\right. & \left.\frac{2}{r}\right) \Gamma_{A B}^{r}-k_{A}^{C} \Gamma_{B C}^{r}-k_{B}^{C} \Gamma_{A C}^{r}+{ }^{(2)} R_{A B}-2^{(2)} D_{B} \beta_{, A} \\
& +\left(\partial_{u}+2 \beta_{, u}+l\right) \Gamma_{A B}^{u}-\Gamma_{u A}^{u} \Gamma_{u B}^{u}-\Gamma_{r A}^{r} \Gamma_{r B}^{r} \\
& -\Gamma_{u A}^{C} \Gamma_{B C}^{u}-\Gamma_{u B}^{C} \Gamma_{A C}^{u}+{ }^{(2)} D_{C}\left(e^{-2 \beta} U^{C} k_{A B}\right) \\
& -e^{-4 \beta} U^{C} k_{B D} U^{D} k_{A C}+2 e^{-2 \beta} \beta_{, C} U^{C} k_{A B},
\end{aligned}
$$


we find

$$
\begin{aligned}
& g^{D A} R_{A B}= e^{-2 \beta}\left[( \partial _ { r } + \frac { 2 } { r } ) \left(l_{B}^{D}+\right.\right. \\
&\left.+k_{B}^{D} \frac{V}{r}+\frac{1}{2}{ }^{(2)} D_{B} U^{D}+\frac{1}{2}{ }^{(2)} D^{D} U_{B}\right) \\
&\left.+k_{A}^{D(2)} D_{B} U^{A}-k_{B}^{A(2)} D_{A} U^{D}+\left(\partial_{u}+l\right) k_{B}^{D}+{ }^{(2)} D_{C}\left(U^{C} k_{B}^{D}\right)\right] \\
&+{ }^{(2)} R_{B}^{D}-2\left({ }^{(2)} D_{B} \partial^{D} \beta+\partial^{D} \beta \partial_{B} \beta+n^{D} n_{B}\right) .
\end{aligned}
$$

When taking into account the previous equations, $G_{u r} \equiv R_{u r}+\frac{1}{2} e^{2 \beta} R=0$ reduces to $g^{A B} R_{A B}=0$. Explicitly, we find from the trace of (4.40)

$$
\begin{gathered}
\partial_{r} V=J, \\
\left.J=e^{2 \beta} r^{2}{ }^{(2)} \Delta \beta+\partial^{D} \beta \partial_{D} \beta+n^{D} n_{D}-\frac{1}{2}{ }^{(2)} R\right)-2 r l-\frac{r^{2}}{2}\left(\partial_{r}+\frac{4}{r}\right)^{(2)} D_{B} U^{B} \\
=-2 r l+e^{2 \beta} r^{2}\left[^{(2)} \Delta \beta+\left(n^{A}-\partial^{A} \beta\right)\left(n_{A}-\partial_{A} \beta\right)\right. \\
\left.-{ }^{(2)} D_{A} n^{A}-\frac{1}{2}{ }^{(2)} R\right]-2 r^{(2)} D_{B} U^{B} \\
=-2 r l-\frac{1}{2} \bar{R}+o\left(r^{-1-\epsilon}\right),
\end{gathered}
$$

where we have used the previous equation to get the second line. This equation implies

$$
\frac{V}{r}=-r l-\frac{1}{2} \bar{R}+r^{-1} 2 M+o\left(r^{-1-\epsilon}\right),
$$

and implies a third arbitrary function of $M\left(u, x^{B}\right)$ as integration constant.

We have $G_{A B}-\frac{1}{2} g_{A B} g^{C D} G_{C D}=R_{A B}-\frac{1}{2} g_{A B} g^{C D} R_{C D}$. Taking into account the previous equations, it thus reduces to the condition that the traceless part of (4.40) vanishes. Using that $\partial_{u} k_{B}^{D}=\partial_{r} l_{B}^{D}-2\left(l_{A}^{D} k_{B}^{A}-k_{A}^{D} l_{B}^{A}\right)$, we get

$$
\begin{aligned}
&\left(\partial_{r}+\frac{1}{r}\right) l_{B}^{D}-\left(l_{A}^{D}\right.\left.k_{B}^{A}-k_{A}^{D} l_{B}^{A}\right)+\frac{1}{2} k_{B}^{D} l= \\
&-\frac{1}{2}\left[\left(\partial_{r}+\frac{2}{r}\right)\left(k_{B}^{D} \frac{V}{r}+\frac{1}{2}{ }^{(2)} D_{B} U^{D}+\frac{1}{2}{ }^{(2)} D^{D} U_{B}\right)\right. \\
&\left.+k_{C}^{D(2)} D_{B} U^{C}-k_{B}^{C(2)} D_{C} U^{D}+{ }^{(2)} D_{C}\left(U^{C} k_{B}^{D}\right)\right] \\
&+e^{2 \beta}\left[n^{D} n_{B}+{ }^{(2)} D_{B} \partial^{D} \beta+\partial^{D} \beta \partial_{B} \beta-\frac{1}{2}{ }^{(2)} R_{B}^{D}\right] .
\end{aligned}
$$

The various definitions then give

$$
\partial_{r} L_{B}^{D}-\frac{1}{r^{2}}\left(L_{A}^{D} K_{B}^{A}-K_{A}^{D} L_{B}^{A}\right)=J_{B}^{D},
$$


where

$$
\begin{aligned}
J_{B}^{D}= & -\frac{r}{2}\left[\left(\partial_{r}+\frac{2}{r}\right)\left(k_{B}^{D} \frac{V}{r}+\frac{1}{2}{ }^{(2)} D_{B} U^{D}+\frac{1}{2}{ }^{(2)} D^{D} U_{B}\right)\right. \\
& \left.+k_{C}^{D(2)} D_{B} U^{C}-k_{B}^{C(2)} D_{C} U^{D}+{ }^{(2)} D_{C}\left(U^{C} k_{B}^{D}\right)\right]+ \\
+ & r e^{2 \beta}\left[n^{D} n_{B}+{ }^{(2)} D_{B} \partial^{D} \beta+\partial^{D} \beta \partial_{B} \beta-\frac{1}{2}{ }^{(2)} R_{B}^{D}\right]-\frac{1}{2} \bar{\gamma}^{D A} \bar{\gamma}_{A B, u}-\frac{r}{2} k_{B}^{D} l \\
& \quad-\frac{1}{2 r}\left(K_{A}^{D} \bar{\gamma}^{A C} \bar{\gamma}_{C B, u}-\bar{\gamma}^{D C} \bar{\gamma}_{C A, u} K_{B}^{A}\right) .
\end{aligned}
$$

The previous equations imply

$$
\begin{gathered}
J_{B}^{D}=-\frac{1}{2}\left(\partial_{r} k_{B}^{D}+\frac{1}{r} k_{B}^{D}\right) V-\frac{r^{2}}{2} k_{B}^{D} e^{2 \beta}\left[{ }^{(2)} \Delta \beta+\left(n^{A}-\partial^{A} \beta\right)\left(n_{A}-\partial_{A} \beta\right)-{ }^{(2)} D_{A} n^{A}-\frac{1}{2}{ }^{(2)} R\right] \\
-\frac{1}{2}\left({ }^{(2)} D_{B} U^{D}+{ }^{(2)} D^{D} U_{B}\right)-r U^{C(2)} D_{C} k_{B}^{D}+\frac{r}{2} k_{C}^{D}\left({ }^{(2)} D^{C} U_{B}-{ }^{(2)} D_{B} U^{C}\right) \\
+\frac{r}{2} k_{B}^{C}\left({ }^{(2)} D_{C} U^{D}-{ }^{(2)} D^{D} U_{C}\right)+\frac{r}{2}{ }^{(2)} D_{C} U^{C} k_{B}^{D} \\
+r e^{2 \beta}\left[\left(n^{D}-\partial^{D} \beta\right)\left(n_{B}-\partial_{B} \beta\right)+{ }^{(2)} D_{B} \partial^{D} \beta-\frac{1}{2}\left({ }^{(2)} R_{B}^{D}+{ }^{(2)} D_{B} n^{D}+{ }^{(2)} D^{D} n_{B}\right)\right] \\
-\frac{1}{2} \bar{\gamma}^{D A} \bar{\gamma}_{A B, u}+\frac{r}{2} k_{B}^{D} l-\frac{1}{2 r}\left(K_{A}^{D} \bar{\gamma}^{A C} \bar{\gamma}_{C B, u}-\bar{\gamma}^{D C} \bar{\gamma}_{C A, u} K_{B}^{A}\right) .
\end{gathered}
$$

Let $\mathcal{O}_{B C}^{D A}=-\frac{1}{r^{2}}\left(K_{C}^{D} \delta_{B}^{A}-\delta_{C}^{D} K_{B}^{A}\right)$ and $\mathcal{A R}$ denote anti-radial ordering. Equation (4.43) without right-hand side has the same form as the Schrödinger equation with time dependent Hamiltonian. If we define

$$
U_{B C}^{D A}\left(r_{<}, r_{>}\right)=\mathcal{A} \mathcal{R} \exp \left[-\int_{r_{<}}^{r_{>}} d r^{\prime} \mathcal{O}_{B C}^{D A}\left(r^{\prime}\right)\right],
$$

the solution to the inhomogeneous equation (4.43) with non-vanishing $J_{D}^{B}$ can then be obtained by variation of constants and reads

$$
L_{B}^{D}(r)=U_{B C}^{D A}(r, \infty)\left[\frac{1}{2} N_{A}^{C}+\int d r^{\prime} U_{A F}^{C E}\left(\infty, r^{\prime}\right) J_{E}^{F}\left(r^{\prime}\right)\right]
$$

and involves two more integration constants encoded in $N_{B}^{D}\left(u, x^{B}\right)$.

In other words, the $r$-dependence of $g_{A B, u}$ is completely determined up to two integration constants. It follows that the only variables left in the theory whose $r$-dependence is undetermined are the two functions contained in $E_{A B}\left(u_{0}, r, x^{C}\right)=g_{A B}\left(u_{0}, r, x^{C}\right)-$ $r^{2} \bar{\gamma}_{A B}\left(u_{0}, x^{C}\right)-r C_{A B}\left(u_{0}, x^{C}\right)-D_{A B}\left(u_{0}, x^{C}\right)-\frac{1}{4} \bar{\gamma}_{A B} C_{D}^{C} C_{C}^{D}$ at some initial fixed $u_{0}$.

When expanding into orders in $r$, one finds in particular

$$
\begin{aligned}
L_{B}^{D} & =\frac{1}{2}\left(\bar{\gamma}^{D A} C_{A B, u}-C^{D A} \bar{\gamma}_{A B, u}\right)+\frac{1}{2} r^{-1}\left[\bar{\gamma}^{D A} \partial_{u}\left(D_{A B}+\frac{1}{4} \bar{\gamma}_{A B} C_{D}^{C} C_{C}^{D}\right)\right. \\
& \left.-C^{D A} C_{A B, u}-D^{D A} \bar{\gamma}_{A B, u}+\frac{1}{4} C_{F}^{E} C_{E}^{F} \bar{\gamma}^{D A} \bar{\gamma}_{A B, u}\right]+o\left(r^{-1-\epsilon}\right), \\
J_{B}^{D} & =\frac{1}{2} \delta_{B}^{D} l-\frac{1}{2} \bar{\gamma}^{D A} \bar{\gamma}_{A B, u}+\frac{1}{4} r^{-1}\left[C^{D A} \bar{\gamma}_{A B, u}-\bar{\gamma}^{D C} \bar{\gamma}_{C A, u} C_{B}^{A}\right] \\
& +\frac{1}{2} r^{-2}\left[l D_{B}^{D}+D^{D A} \bar{\gamma}_{A B, u}-\bar{\gamma}^{D C} \bar{\gamma}_{C A, u} D_{B}^{A}\right]+o\left(r^{-2+\epsilon}\right) .
\end{aligned}
$$


When injecting into the equation of motion (4.43), the leading order requires that

$$
\bar{\gamma}_{A B, u}=l \bar{\gamma}_{A B},
$$

or, in other words, that the only $u$ dependence in $\bar{\gamma}_{A B}$ is contained in the conformal factor. This agrees with the assumption of section 4.1. where the $u$-dependence of $\bar{\gamma}_{A B}$ was contained in $\exp 2 \varphi$ and $l=2 \partial_{u} \varphi$, and also with the discussion at the end of the previous subsection, where it was contained in $\exp 2 \widetilde{\varphi}$ and $l=2 \partial_{u} \widetilde{\varphi}$. In the following we always assume that (4.47) holds. In particular, this implies

$$
\begin{aligned}
L_{B}^{D} & =\frac{1}{2}\left(\bar{\gamma}^{D A} C_{A B, u}-l C_{B}^{D}\right)+\frac{1}{2} r^{-1}\left[\bar{\gamma}^{D A} D_{A B, u}-C^{D A} C_{A B, u}\right. \\
& \left.-l D_{B}^{D}+\frac{1}{2} C^{E F} \partial_{u} C_{E F} \delta_{B}^{D}\right]+o\left(r^{-1-\epsilon}\right), \\
J_{B}^{D} & =\frac{1}{2} r^{-2} l D_{B}^{D}+o\left(r^{-2+\epsilon}\right) .
\end{aligned}
$$

When taking into account the next order of (4.43) and comparing to the general solution (4.46), we get

$$
\partial_{u} D_{A B}=0, \quad N_{A B}=\partial_{u} C_{A B}-C_{A B} l,
$$

where the index on $N_{B}^{A}$ has been lowered with $\bar{\gamma}_{A C}$. This implies in turn that

$$
l_{B}^{A}=\frac{1}{2} l \delta_{B}^{A}+\frac{1}{2} r^{-1} N_{B}^{A}-\frac{1}{4} r^{-2}\left[C_{C}^{A} N_{B}^{C}-N_{C}^{A} C_{B}^{C}+2 l D_{B}^{A}\right]+o\left(r^{-2-\epsilon}\right) .
$$

At this stage, equations (4.29) have been solved, and then (4.31) holds automatically on account of the Bianchi identities. Furthermore $g^{C D} G_{C D}=0$ reduces to $R_{u r}=0$ and we also have $R=0$. Under these assumptions, we only need to discuss the $r$ independent part of $r^{2} G_{u A}=0$ and then of $r^{2} G_{u u}=0$, which reduce to $r^{2} R_{u A}=0$ and $r^{2} R_{u u}=0$, respectively. The $r$-independent part fixes the $u$ dependence of $N_{A}$ and $M$ in terms of the other fields. Explicitly,

$$
\begin{gathered}
R_{u A}=\left(-\partial_{u}+l\right) \beta_{, A}-\partial_{A} l-\left(\partial_{u}+l\right) n_{A} \\
+n_{B}{ }^{(2)} D^{B} U_{A}-\beta_{, B}{ }^{(2)} D_{A} U^{B}+2 U^{B}\left(\beta_{, B} \beta_{, A}+n_{B} n_{A}\right) \\
+{ }^{(2)} D_{B}\left[l_{A}^{B}+\frac{1}{2}{ }^{(2)} D^{B} U_{A}-\frac{1}{2}{ }^{(2)} D_{A} U^{B}+U^{B}\left(\beta, A-n_{A}\right)\right]+2 n_{B} l_{A}^{B} \\
-\left(\partial_{r}+2 \beta_{, r}+\frac{2}{r}\right)\left(\frac{V_{, A}}{2 r}\right)-\frac{V}{r}\left(\partial_{r}+\frac{2}{r}\right) n_{A}+k_{A}^{B}\left(\frac{V_{, B}}{r}+2 \frac{V}{r} n_{B}\right) \\
-e^{-2 \beta}\left(\partial_{r}+\frac{2}{r}\right)\left[U^{B}\left(\frac{1}{2}{ }^{(2)} D_{A} U_{B}+\frac{1}{2}{ }^{(2)} D_{B} U_{A}+l_{A B}+\frac{V}{r} k_{A B}\right)\right] \\
-e^{-2 \beta} U^{B}\left[\left(\partial_{u}+l\right) k_{A B}-2 l_{A}^{C} k_{C B}-2 k_{A}^{C} l_{C B}-2 k_{A}^{C} k_{C B} \frac{V}{r}\right. \\
\left.\quad+{ }^{(2)} D_{C}\left(k_{A B} U^{C}\right)-k_{A C}{ }^{(2)} D^{C} U_{B}-k_{B C}{ }^{(2)} D^{C} U_{A}\right]
\end{gathered}
$$


and the term proportional to $r^{-2}$ yields

$$
\begin{aligned}
&\left(\partial_{u}+l\right) N_{A}=\partial_{A} M+\frac{1}{4} C_{A}^{B} \partial_{B} \bar{R}+\frac{1}{16} \partial_{A}\left[N_{C}^{B} C_{B}^{C}\right]-\frac{1}{4} \bar{D}_{A} C_{B}^{C} N_{C}^{B} \\
&-\frac{1}{4} \bar{D}_{B}\left[C_{C}^{B} N_{A}^{C}-N_{C}^{B} C_{A}^{C}\right]-\frac{1}{4} \bar{D}_{B}\left[\bar{D}^{B} \bar{D}_{C} C_{A}^{C}-\bar{D}_{A} \bar{D}_{C} C^{B C}\right] \\
& \quad-\frac{1}{32} l \partial_{A}\left[C_{C}^{B} C_{B}^{C}\right]+\frac{1}{16} \partial_{A} l C_{C}^{B} C_{B}^{C}+\frac{1}{2} \bar{D}_{B}\left[l D_{A}^{B}\right] .
\end{aligned}
$$

Similarily,

$$
\begin{aligned}
R_{u u}=\left(\partial_{u}+2 \beta_{, u}+l\right) \Gamma_{u u}^{u}+\left(\partial_{r}+2 \beta_{, r}+\frac{2}{r}\right) \Gamma_{u u}^{r}+\left(\partial_{A}+2 \beta_{, A}+{ }^{(2)} \Gamma_{B A}^{B}\right) \Gamma_{u u}^{A} \\
-2 \beta_{, u u}-\partial_{u} l-\left(\Gamma_{u u}^{u}\right)^{2}-2 \Gamma_{u A}^{u} \Gamma_{u u}^{A}-\left(\Gamma_{u r}^{r}\right)^{2}-2 \Gamma_{u A}^{r} \Gamma_{u r}^{A}-\Gamma_{u B}^{A} \Gamma_{u A}^{B},
\end{aligned}
$$

and the term proportional to $r^{-2}$ yields

$$
\begin{aligned}
\left(\partial_{u}+\frac{3}{2} l\right) M=-\frac{1}{8} N_{B}^{A} N_{A}^{B} & -\frac{1}{8} l C_{B}^{A} N_{A}^{B}-\frac{1}{32} l^{2} C_{B}^{A} C_{A}^{B}+\frac{1}{8} \bar{\Delta} \bar{R} \\
& +\frac{1}{4} \bar{D}_{A} \bar{D}_{C} N^{C A}+\frac{1}{8} l \bar{D}_{A} \bar{D}_{C} C^{C A}+\frac{1}{4} \bar{D}_{C} l \bar{D}_{A} C^{C A}
\end{aligned}
$$

All these considerations can be summarized as follows:

For a metric of the form (4.1) satisfying the determinant condition and with $g_{A B}$ as in (4.35), the general solution to Einstein's equations is parametrized by the 2 dimensional background metric $\bar{\gamma}_{A B}\left(u, x^{C}\right)$ satisfying (4.47), by the mass and angular momentum aspects $M\left(u, x^{A}\right), N_{A}\left(u, x^{B}\right)$ satisfying (4.50), (4.49), by the traceless symmetric news tensor $N_{A B}\left(u, x^{C}\right)$ defined in (4.48), and by the traceless symmetric tensors $D_{A B}\left(x^{C}\right)$, $C_{A B}\left(u_{0}, x^{C}\right), E_{A B}\left(u_{0}, r, x^{C}\right)$.

For such spacetimes, the only non vanishing components of the unphysical Weyl tensor at the boundary are given by (4.38). When logarithmic terms are required to be absent in the metric, $D_{A B}\left(x^{C}\right)$ has to satisfy $\bar{D}_{B} D_{A}^{B}=0$. In the coordinates $\zeta, \bar{\zeta}$ and the parametrization $\bar{\gamma}_{A B} d x^{A} d x^{B}=e^{2 \widetilde{\varphi}} d \zeta d \bar{\zeta}$, this leads to (4.39) with $d=d(\zeta)$ and $\bar{d}=\bar{d}(\bar{\zeta})$ by also taking (4.48) into account.

In particular, let us now use the parametrization $\bar{\gamma}_{A B} d x^{A} d x^{B}=e^{2 \widetilde{\varphi}} d \zeta d \bar{\zeta}$. The determinant condition then reads $\operatorname{det} g_{A B}=e^{4 \widetilde{\varphi}} \frac{r^{4}}{4}$. Even though we will not use it explicitly below, let us point out that the determinant condition can be implemented for instance by choosing the Beltrami representation,

$$
\begin{gathered}
h=\frac{g_{\zeta \zeta}}{g_{\zeta \bar{\zeta}}+f}, \quad \bar{h}=\frac{g_{\bar{\zeta} \bar{\zeta}}}{g_{\zeta \bar{\zeta}}+f} \\
g_{\zeta \zeta}=\frac{2 f h}{1-y}, \quad g_{\overline{\zeta \zeta}}=\frac{2 f \bar{h}}{1-y}, \quad g_{\zeta \bar{\zeta}}=\frac{f(1+y)}{1-y}, \\
g^{\zeta \zeta}=-\frac{2 \bar{h}}{f(1-y)}, \quad g^{\overline{\zeta \zeta}}=-\frac{2 h}{f(1-y)}, \quad g^{\zeta \bar{\zeta}}=\frac{1+y}{f(1-y)},
\end{gathered}
$$


where $f=\sqrt{-^{(2)} g}, y=h \bar{h}$, with $f=\frac{r^{2}}{2} e^{2 \widetilde{\varphi}}$ fixed, while $h=O\left(r^{-1}\right)=\bar{h}$. Alternatively, one can choose

$$
\begin{gathered}
g_{\zeta \zeta}=f e^{i \alpha} \sinh \rho, \quad g_{\overline{\zeta \zeta} \bar{\zeta}}=f e^{-i \alpha} \sinh \rho, \quad g_{\zeta \bar{\zeta}}=f \cosh \rho, \\
g^{\zeta \zeta}=-f^{-1} e^{-i \alpha} \sinh \rho, \quad g^{\bar{\zeta} \bar{\zeta}}=-f^{-1} e^{i \alpha} \sinh \rho, \quad g^{\zeta \bar{\zeta}}=f^{-1} \cosh \rho,
\end{gathered}
$$

where $\rho=O\left(r^{-1}\right)$ and $\alpha=O\left(r^{0}\right)$.

In the parametrization with the conformal factor introduced with respect to the Riemann sphere, we can write

$$
\begin{gathered}
C_{\zeta \zeta}=e^{2 \widetilde{\varphi}} c, \quad C_{\bar{\zeta} \bar{\zeta}}=e^{2 \widetilde{\varphi}} \bar{c}, \quad C_{\zeta \bar{\zeta}}=0, \\
D_{\zeta \zeta}=d, \quad D_{\overline{\zeta \zeta}}=\bar{d}, \quad D_{\zeta \bar{\zeta}}=0 .
\end{gathered}
$$

Equations (4.36), (4.37) and 4.42) read

$$
\begin{aligned}
& \beta=-\frac{1}{4} r^{-2} c \bar{c}-\frac{1}{3} r^{-3} e^{-2 \widetilde{\varphi}}(d \bar{c}+\bar{d} c)+o\left(r^{-3-\epsilon}\right), \\
& U^{\zeta}=-\frac{2}{r^{2}} e^{-4 \widetilde{\varphi}} \partial\left(e^{2 \widetilde{\varphi}} \bar{c}\right)- \quad-\frac{2}{3 r^{3}}\left[\left(\ln r+\frac{1}{3}\right) 4 e^{-4 \widetilde{\varphi}} \partial \bar{d}-4 e^{-4 \widetilde{\varphi}} \bar{c} \bar{\partial}\left(e^{2 \widetilde{\varphi}} c\right)+N^{\zeta}\right]+o\left(r^{-3-\epsilon}\right), \\
& \frac{V}{r}=-2 r \partial_{u} \widetilde{\varphi}+4 e^{-2 \widetilde{\varphi}} \partial \bar{\partial} \widetilde{\varphi}+r^{-1} 2 M+o\left(r^{-1-\epsilon}\right) .
\end{aligned}
$$

and the evolution equations become

$$
\begin{aligned}
& \partial_{u}\left(e^{3 \widetilde{\varphi}} M\right)=\partial_{u}\left(e^{\widetilde{\varphi}}\left[\partial^{2} \bar{c}+\bar{\partial}^{2} c+2 \partial \widetilde{\varphi} \partial \bar{c}+2 \bar{\partial} \widetilde{\varphi} \bar{\partial} c+2 \partial^{2} \widetilde{\varphi} \bar{c}+2 \bar{\partial}^{2} \widetilde{\varphi} c\right]\right) \\
& -e^{\widetilde{\varphi}} \partial_{u}\left(e^{\widetilde{\varphi}} c\right) \partial_{u}\left(e^{\widetilde{\varphi}} \bar{c}\right)+2 e^{\widetilde{\varphi}}\left(c\left[\partial_{u}(\bar{\partial} \widetilde{\varphi})^{2}-\partial_{u} \bar{\partial}^{2} \widetilde{\varphi}\right]+\bar{c}\left[\partial_{u}(\partial \widetilde{\varphi})^{2}-\partial_{u} \partial^{2} \widetilde{\varphi}\right]\right) \\
& +e^{-\widetilde{\varphi}}\left(-4(\partial \bar{\partial})^{2} \widetilde{\varphi}+8\left[(\partial \bar{\partial} \widetilde{\varphi})^{2}+\partial \widetilde{\varphi} \partial \bar{\partial}^{2} \widetilde{\varphi}+\bar{\partial} \widetilde{\varphi} \partial^{2} \bar{\partial} \widetilde{\varphi}-2 \bar{\partial} \widetilde{\varphi} \partial \widetilde{\varphi} \partial \bar{\partial} \widetilde{\varphi}\right]\right), \\
& \partial_{u}\left(e^{2 \widetilde{\varphi}} N_{\bar{\zeta}}\right)=e^{2 \widetilde{\varphi}}\left[\bar{\partial} M+\frac{1}{4}\left[(\bar{\partial} \bar{c}+5 \bar{c} \bar{\partial}) \partial_{u} c-(3 c \bar{\partial}+7 \bar{\partial} c) \partial_{u} \bar{c}\right]+2 \bar{\partial} \widetilde{\varphi}\left(\bar{c} \partial_{u} c-c \partial_{u} \bar{c}\right)\right. \\
& \left.-\frac{1}{2} \partial_{u} \widetilde{\varphi} \bar{\partial}(c \bar{c})+\bar{\partial} \partial_{u} \widetilde{\varphi} c \bar{c}\right]+2\left(\partial \partial_{u} \widetilde{\varphi}+\partial_{u} \widetilde{\varphi} \partial\right) \bar{d} \\
& +\bar{\partial}^{3} c+2 \bar{\partial}^{3} \widetilde{\varphi} c+4 \bar{\partial}^{2} \widetilde{\varphi} \bar{\partial} c-4 \bar{\partial} \widetilde{\varphi} \bar{\partial}^{2} \widetilde{\varphi} c-4(\bar{\partial} \widetilde{\varphi})^{2} \bar{\partial} c \\
& -\partial^{2} \bar{\partial} \bar{c}-2\left(\partial \widetilde{\varphi} \partial+\partial^{2} \widetilde{\varphi}\right) \bar{\partial} \bar{c}-2(\partial \bar{\partial} \widetilde{\varphi}-\bar{\partial} \widetilde{\varphi} \partial-2 \bar{\partial} \widetilde{\varphi} \partial \widetilde{\varphi}) \partial \bar{c} \\
& -2\left(\partial^{2} \bar{\partial} \widetilde{\varphi}+2 \partial \bar{\partial}^{2} \widetilde{\varphi}-2 \bar{\partial} \widetilde{\varphi} \partial^{2} \widetilde{\varphi}-4 \bar{\partial} \widetilde{\varphi} \partial \bar{\partial} \widetilde{\varphi}\right) \bar{c} .
\end{aligned}
$$

Let us now set $\widetilde{\varphi}=0$. Note that one can re-introduce an arbitrary $\widetilde{\varphi}$ through the finite coordinate transformation generated by $\xi^{u}=-u \widetilde{\varphi}, \xi^{A}=-\xi_{, B}^{u} \int_{r}^{\infty} d r^{\prime}\left(e^{2 \beta} g^{A B}\right)$, 


$$
\begin{aligned}
\xi^{r}=-\frac{1}{2} r\left(\partial_{A} \xi^{A}-2 \widetilde{\varphi}-f_{, B} U^{B}\right) . \text { The above relations then simplify to } \\
\beta=-\frac{1}{4} r^{-2} c \bar{c}-\frac{1}{3} r^{-3}(d \bar{c}+\bar{d} c)+o\left(r^{-3-\epsilon}\right), \\
U^{\zeta}=-2 r^{-2} \partial \bar{c}-\frac{2}{3} r^{-3}\left[\left(\ln r+\frac{1}{3}\right) 4 \partial \bar{d}-4 \bar{c} \bar{\partial} c+N^{\zeta}\right]+o\left(r^{-3-\epsilon}\right), \\
\frac{V}{r}=r^{-1} 2 M+o\left(r^{-1-\epsilon}\right), \\
\partial_{u} M=\left[\partial^{2} \overline{\bar{c}}+\bar{\partial}^{2} \dot{c}\right]-\dot{\bar{c}} \overline{\bar{c}} \\
\partial_{u} N_{\bar{\zeta}}=\bar{\partial} M+\frac{1}{4}[(\bar{\partial} \bar{c}+5 \bar{c} \bar{\partial}) \dot{c}-(3 c \bar{\partial}+7 \bar{\partial} c) \dot{\bar{c}}]+\bar{\partial}^{3} c-\partial^{2} \bar{\partial} \bar{c} .
\end{aligned}
$$

When defining $\widetilde{M}=M-\bar{\partial}^{2} c-\partial^{2} \bar{c}$ and $\widetilde{N}^{\zeta}=-\frac{1}{12}\left[2 N^{\zeta}+7 \bar{c} \bar{\partial} c+3 c \bar{\partial} \bar{c}\right]$, the evolution equations become

$$
\begin{aligned}
\partial_{u} \widetilde{M} & =-\dot{\bar{c}} \overline{\bar{c}} \\
3 \partial_{u} \widetilde{N^{\zeta}} & =-\bar{\partial} \widetilde{M}-2 \bar{\partial}^{3} c-(\bar{\partial} \bar{c}+3 \bar{c} \bar{\partial}) \dot{c} .
\end{aligned}
$$

\subsection{Realization of $\mathfrak{b m s}_{4}$ on solution space}

In order to compute how $\mathfrak{b m s}_{4}$ is realized on solution space we need to compute the Lie derivative of the metric on-shell. We will do so for the extended transformations defined by (4.26)-(4.27) and use $-\delta \bar{\gamma}_{A B}=2 \omega \bar{\gamma}_{A B}$. Let $\widetilde{\psi}=\psi-2 \omega$. This gives

$$
-\delta C_{A B}=\left[f \partial_{u}+\mathcal{L}_{Y}-\frac{1}{2}(\widetilde{\psi}+f l)\right] C_{A B}-2 \bar{D}_{A} \bar{D}_{B} f+\bar{\Delta} f \bar{\gamma}_{A B},
$$

where (4.48) should be used to eliminate $\partial_{u} C_{A B}$ in favor of $N_{A B}$ and

$$
-\delta D_{A B}=\mathcal{L}_{Y} D_{A B},
$$

where we have used that

$$
\begin{array}{r}
\bar{D}_{A} \bar{D}_{C} f C_{B}^{C}+\bar{D}_{B} \bar{D}_{C} f C_{A}^{C}-\bar{\gamma}_{A B} \bar{D}_{C} \bar{D}_{C} f C^{C D}-\bar{\Delta} f C_{A B}=0, \\
\bar{D}_{A} f \bar{D}_{C} C_{B}^{C}+\bar{D}_{B} f \bar{D}_{C} C_{A}^{C}+\bar{D}_{C} f \bar{D}_{A} C_{B}^{C}+\bar{D}_{C} f \bar{D}_{B} C_{A}^{C}- \\
-\bar{D}^{C} f \bar{D}_{C} C_{A B}-\bar{\gamma}_{A B} \bar{D}_{C} f \bar{D}_{D} C^{C D}=0,
\end{array}
$$

which can be explicitly checked in the parametrization $\bar{\gamma}_{A B} d x^{A} d x^{B}=e^{2 \widetilde{\varphi}} d \zeta d \bar{\zeta}$ with $C_{A B}$ defined in (4.51). By taking the time derivative of (4.56) and using (4.48), (4.9) with $\psi$ replaced by $\widetilde{\psi}$, one finds the transformation law for the news tensor,

$$
\begin{aligned}
-\delta N_{A B}=\left[f \partial_{u}+\mathcal{L}_{Y}\right] & N_{A B}-\left(\bar{D}_{A} \bar{D}_{B} \widetilde{\psi}-\frac{1}{2} \bar{\Delta} \widetilde{\psi} \bar{\gamma}_{A B}\right) \\
& +\frac{1}{4}\left(2 f i+f l^{2}+\widetilde{\psi} l-4 \dot{\omega}+2 Y^{C} \bar{D}_{C} l\right) C_{A B} \\
& \quad+l\left(\bar{D}_{A} \bar{D}_{B} f-\frac{1}{2} \bar{\Delta} f \bar{\gamma}_{A B}\right)-f\left(\bar{D}_{A} \bar{D}_{B} l-\frac{1}{2} \bar{\Delta} l \bar{\gamma}_{A B}\right) .
\end{aligned}
$$


We have $g_{u A}=\frac{1}{2} \bar{D}_{B} C_{A}^{B}+\frac{2}{3} r^{-1}\left[\left(\ln r+\frac{1}{3}\right) \bar{D}_{B} D_{A}^{B}+\frac{1}{4} C_{A}^{B} \bar{D}_{C} C_{B}^{C}+N_{A}\right]+o\left(r^{-1-\epsilon}\right)$, and by computing $\mathcal{L}_{\xi} g_{u A}$ on-shell, we find to leading oder that $-\delta\left(\bar{D}_{B} C_{A}^{B}\right)=\left[f \partial_{u}+\right.$ $\left.\mathcal{L}_{Y}+\frac{1}{2}(l f+\widetilde{\psi})\right] \bar{D}_{B} C_{A}^{B}-\frac{1}{2} \partial_{B}(l f+\widetilde{\psi}) C_{A}^{B}+\partial_{C} f\left(N_{A}^{C}+l C_{A}^{C}\right)-\partial_{A}(\bar{\Delta} f)-\partial_{A} f \bar{R}$. This is consistent with (4.56) by using the generalization of (4.10) which reads

$$
2 \bar{D}_{B} \bar{D}_{C} Y_{A}=\bar{\gamma}_{C A} \bar{D}_{B} \psi+\bar{\gamma}_{A B} \bar{D}_{C} \psi-\bar{\gamma}_{B C} \bar{D}_{A} \psi+\bar{R} Y_{C} \bar{\gamma}_{B A}-\bar{R} Y_{A} \bar{\gamma}_{B C},
$$

and implies $\bar{\Delta} Y^{A}=-\frac{1}{2} \bar{R} Y^{A}, \bar{\Delta} \psi=-\bar{R} \psi$. The logarithmic term gives $-\delta\left(\bar{D}_{B} D_{A}^{B}\right)=$ $\left(f \partial_{u}+\mathcal{L}_{Y}+l f+\widetilde{\psi}\right) \bar{D}_{B} D_{A}^{B}$, which is again consistent with (4.57), while the $r^{-1}$ terms, when combined with the previous transformations, give

$$
\begin{gathered}
-\delta N_{A}=\left[f \partial_{u}+\mathcal{L}_{Y}+\widetilde{\psi}+f l\right] N_{A}-\frac{1}{2}\left[f \bar{D}_{B} l+\bar{D}_{B} \widetilde{\psi}+(\widetilde{\psi}+l f) \bar{D}_{B}\right] D_{A}^{B} \\
+3 \bar{D}_{A} f M-\frac{3}{16} \bar{D}_{A} f N_{C}^{B} C_{B}^{C}+\frac{1}{2} \bar{D}_{B} f N_{C}^{B} C_{A}^{C}+\frac{1}{32}\left(\bar{D}_{A} f l-f \bar{D}_{A} l-\bar{D}_{A} \widetilde{\psi}\right)\left(C_{C}^{B} C_{B}^{C}\right) \\
+\frac{1}{4}\left(\bar{D}_{B} f \bar{R}+\bar{D}_{B} \bar{\Delta} f\right) C_{A}^{B}-\frac{3}{4} \bar{D}_{B} f\left(\bar{D}^{B} \bar{D}_{C} C_{A}^{C}-\bar{D}_{A} \bar{D}_{C} C^{B C}\right) \\
\quad+\frac{1}{2}\left(\bar{D}_{A} \bar{D}_{B} f-\frac{1}{2} \bar{\Delta} f \bar{\gamma}_{A B}\right) \bar{D}_{C} C^{C B}+\frac{3}{8} \bar{D}_{A}\left(\bar{D}_{C} \bar{D}_{B} f C^{C B}\right) .
\end{gathered}
$$

Here $\partial_{u} N_{A}$ should be eliminated by using (4.49). In the same way, from the order $r^{-1}$ of $\mathcal{L}_{\xi} g_{u u}$, we get

$$
\begin{aligned}
& -\delta M=\left[f \partial_{u}+Y^{A} \partial_{A}+\frac{3}{2}(\widetilde{\psi}+f l)\right] M \\
& +\frac{1}{4} \partial_{u}\left[\bar{D}_{C} \bar{D}_{B} f C^{C B}+2 \bar{D}_{B} f \bar{D}_{C} C^{C B}\right]+\frac{1}{4}\left[\bar{D}_{A} f l-f \bar{D}_{A} l-\bar{D}_{A} \widetilde{\psi}\right] \bar{D}_{B} C^{B A} \\
& \quad+\frac{1}{4} \partial_{A} f\left(\partial^{A} \bar{R}-C^{A B} \bar{D}_{B} l\right)+\frac{1}{4} l\left[\bar{D}_{C} \bar{D}_{B} f C^{C B}+\bar{D}_{B} f \bar{D}_{C} C^{C B}\right],
\end{aligned}
$$

where $\partial_{u} M$ should be replaced by its expression from (4.50).

Let us now discuss these transformations in the parametrization $\zeta, \bar{\zeta}$ with $\widetilde{\varphi}=0=\omega$ so that $\bar{\gamma}_{A B} d x^{A} d x^{B}=d \zeta d \bar{\zeta}$. From the leading and subleading orders of $\mathcal{L}_{\xi} g_{\zeta \zeta}, \mathcal{L}_{\xi} g_{\bar{\zeta} \bar{\zeta}}$, we get

$$
\begin{gathered}
-\delta c=f \dot{c}+Y^{A} \partial_{A} c+\left(\frac{3}{2} \partial Y-\frac{1}{2} \bar{\partial} \bar{Y}\right) c-2 \partial^{2} f, \\
-\delta d=Y^{A} \partial_{A} d+2 \partial Y d,
\end{gathered}
$$

with $f$ given in (4.24) and the complex conjugate relation holding for $\bar{c}, \bar{d}$. In particular, for the news function we find

$$
-\delta \dot{c}=f \ddot{c}+Y^{A} \partial_{A} \dot{c}+2 \partial Y \dot{c}-\partial^{3} Y,
$$

From the subleading term of $\mathcal{L}_{\xi} g^{r \zeta}$ and the leading term of $\mathcal{L}_{\xi} g_{u u}$ and we get

$$
\begin{aligned}
-\delta \widetilde{N}^{\zeta}=Y^{A} \partial_{A} \widetilde{N}^{\zeta} & +(\partial Y+2 \bar{\partial} \bar{Y}) \widetilde{N}^{\zeta}+\frac{1}{3} \partial(\psi \bar{d}) \\
& -\bar{\partial} f\left(\widetilde{M}+2 \bar{\partial}^{2} c+\bar{c} \dot{c}\right)-\frac{f}{3}\left[\bar{\partial} \widetilde{M}+2 \bar{\partial}^{3} c+(\bar{\partial} \bar{c}+3 \bar{c} \bar{\partial}) \dot{c}\right]
\end{aligned}
$$




$$
-\delta \widetilde{M}=-f \dot{\bar{c}} \dot{\bar{c}}+Y^{A} \partial_{A} \widetilde{M}+\frac{3}{2} \psi \widetilde{M}+\bar{c} \partial^{3} Y+c \bar{\partial}^{3} \bar{Y}+4 \partial^{2} \bar{\partial}^{2} \widetilde{T} .
$$

As can be understood by comparing with the 3 dimensional anti-de Sitter and flat cases, this computation already contains information on the central extensions in the surface charge algebra through the inhomogeneous part of the transformation laws for the fields because the normalization of the generators is known from the charges of the Kerr solution. We plan to return to the details of this question elsewhere.

\section{Conclusion and outlook}

In this work, we have shown that the symmetry algebra of asymptotically flat 4 dimensional spacetimes is $\mathfrak{b m s}_{4}$, an algebra that contains both the Poincaré algebra and the non centrally extended Virasoro algebra in a completely natural way. As a first non trivial effect, we have computed the transformation properties of the data characterizing solution space.

We have not analyzed in detail the singularities of the $\mathfrak{b m s}_{4}$ transformations nor those of the classical solutions that ought to be allowed. Indeed, in the Bondi-Metzner-Sachs gauge, the $\mathfrak{b m s}_{4}$ transformations that we are advocationg involve singularities at two points of the sphere at infinity that extend to $\Im^{+}$or to $\Im^{-}$and into the bulk. The reason why we have choosen this approach to asymptotically flat spacetimes at null infinity is its similarity with the Fefferman-Grahan definition of asymptotically anti-de Sitter spacetimes. The appearance of the extended transformations does not depend on this choice however. Indeed, in the geometrical approach to asymptotic flatness as developed in [64], there is also room for the extended transformations since the quotient algebra of the asymptotic symmetry algebra by the infinitesimal supertranslations is again characterized by the conformal Killing vectors of the two-sphere.

More to the point, one can also consider a definition of asymptotic flatness in 4 dimensions at null infinity that does not completely fix the gauge, as done for instance in [3] for asymptotically $A d S_{3}$ spacetimes. Such conditions can be inferred for instance from equation (11.1.24) of [65]. In this case, $\mathfrak{b m s}_{4}$ appears as the quotient algebra of allowed transformations modulo trivial ones. By adapting the arguments of footnote 6 of [13] one can then improve the asymptotic symmetry generators through pure gauge transformations in such a way as to remove all singularities in the asymptotic symmetry generators at finite radius. From this point of view, the bulk singularities of the asymptotic symmetry generators appear as an artefact of the Bondi-Metzner-Sachs gauge fixation.

We believe that our understanding of the symmetry structure and its action on solution space goes some way in getting quantitative control on "structure $X$ " [66], i.e., on a holographic description of gravity with zero cosmological constant. 
What we plan to do next is a systematic discussion of the central extensions and the representation theory of $\mathfrak{b m s}_{4}$ on the one hand, and a construction of the associated algebra of surface charges and generators on the other. In the future, it should be interesting to analyze in more details the consequences of our results on local conformal invariance for the non extremal Kerr/CFT correspondence and for the gravitational S-matrix for instance.

\section{Acknowledgements}

The authors thank M. Bañados, G. Compère, G. Giribet, A. Gomberoff, M. Henneaux, A. Kleinschmidt, C. Martínez, R. Troncoso and A. Virmani for useful discussions. This work is supported in parts by the Fund for Scientific Research-FNRS (Belgium), by the Belgian Federal Science Policy Office through the Interuniversity Attraction Pole P6/11, by IISN-Belgium and by Fondecyt projects No. 1085322 and No. 1090753.

\section{References}

[1] J. M. Maldacena, "The large N limit of superconformal field theories and supergravity," Adv. Theor. Math. Phys. 2 (1998) 231-252, hep-th/9711200.

[2] M. Henneaux and C. Teitelboim, "Asymptotically anti-de Sitter spaces," Commun. Math. Phys. 98 (1985) 391.

[3] J. D. Brown and M. Henneaux, "Central charges in the canonical realization of asymptotic symmetries: An example from three-dimensional gravity," Commun. Math. Phys. 104 (1986) 207.

[4] M. Henneaux, "Asymptotically anti-de Sitter universes in $\mathrm{d}=3,4$ and higher dimensions," in Proceedings of the Fourth Marcel Grossmann Meeting on General Relativity, Rome 1985, R. Ruffini, ed., pp. 959-966. Elsevier Science Publishers B.V., 1986.

[5] H. Bondi, M. G. van der Burg, and A. W. Metzner, "Gravitational waves in general relativity. 7. Waves from axisymmetric isolated systems," Proc. Roy. Soc. Lond. A 269 (1962) 21.

[6] R. K. Sachs, "Gravitational waves in general relativity. 8. Waves in asymptotically flat space-times," Proc. Roy. Soc. Lond. A 270 (1962) 103.

[7] R. K. Sachs, "Asymptotic symmetries in gravitational theories," Phys. Rev. 128 (1962) 2851-2864. 
[8] G. Barnich and C. Troessaert, "Symmetries of asymptotically flat 4 dimensional spacetimes at null infinity revisited," 0909.2617.

[9] G. Barnich and G. Compère, "Classical central extension for asymptotic symmetries at null infinity in three spacetime dimensions," Class. Quant. Grav. 24 (2007) F15, gr-qc/0610130. Corrigendum: ibid 24 (2007) 3139.

[10] A. Ashtekar, J. Bicak, and B. G. Schmidt, "Behavior of Einstein-Rosen waves at null infinity," Phys. Rev. $D 55$ (1997) 687-694, gr-qc/9608041.

[11] A. Ashtekar, J. Bicak, and B. G. Schmidt, "Asymptotic structure of symmetry reduced general relativity,” Phys. Rev. D55 (1997) 669-686, gr-qc/9608042.

[12] A. Strominger, "Black hole entropy from near-horizon microstates," JHEP 02 (1998) 009, arXiv: hep-th/9712251.

[13] M. Guica, T. Hartman, W. Song, and A. Strominger, "The Kerr/CFT Correspondence," Phys. Rev. D80 (2009) 124008, 0809.4266.

[14] S. Carlip, "The statistical mechanics of the (2+1)-dimensional black hole," Phys. Rev. D51 (1995) 632-637, gr-qc/9409052.

[15] S. N. Solodukhin, “Conformal description of horizon's states," Phys. Lett. B454 (1999) 213-222, hep-th/9812056.

[16] S. Carlip, "Black hole entropy from conformal field theory in any dimension," Phys. Rev. Lett. 82 (1999) 2828-2831, hep-th/9812013.

[17] S. Carlip, "Entropy from conformal field theory at Killing horizons," Class. Quant. Grav. 16 (1999) 3327-3348, gr-qc/9906126.

[18] M. Park and J. Ho, "Comments on 'Black hole entropy from conformal field theory in any dimension'," Phys. Rev. Lett. 83 (1999) 5595, hep-th/9910158.

[19] M.-I. Park, "Hamiltonian dynamics of bounded spacetime and black hole entropy: Canonical method," Nucl. Phys. B634 (2002) 339-369, hep-th/0111224.

[20] I. Sachs and S. N. Solodukhin, "Horizon holography," Phys. Rev. D64 (2001) 124023, hep-th/0107173.

[21] J. Koga, "Asymptotic symmetries on Killing horizons," Phys. Rev. D64 (2001) 124012, gr-qc/0107096.

[22] S. Carlip, "Near-horizon conformal symmetry and black hole entropy," Phys. Rev. Lett. 88 (2002) 241301, gr-qc/0203001. 
[23] S. Silva, "Black hole entropy and thermodynamics from symmetries," Class. Quant. Grav. 19 (2002) 3947-3962, hep-th/0204179.

[24] G. Kang, J. Koga, and M. Park, "Near-horizon conformal symmetry and black hole entropy in any dimension," Phys. Rev. D70 (2004) 024005, hep-th / 0402113.

[25] J. Koga, "Universal properties from local geometric structure of Killing horizon," Class. Quant. Grav. 24 (2007) 3067-3084, gr-qc/0604054.

[26] J. Koga, "Asymptotic symmetries on Kerr-Newman horizon without anomaly of diffeomorphism invariance," Class. Quant. Grav. 25 (2008) 045009, gr-qc/0609120.

[27] A. Ashtekar, "Asymptotic quantization of the gravitational field," Phys. Rev. Lett. 46 (1981) 573-576.

[28] A. Ashtekar, “Asymptotic Quantization: Based on 1984 Naples Lectures,”. Naples, Italy: Bibliopolis (1987) 107 p. (Monographs and textbooks in physical science, 2).

[29] S. Hollands and A. Ishibashi, "Asymptotic flatness and Bondi energy in higher dimensional gravity," J. Math. Phys. 46 (2005) 022503, gr-qc/0304054.

[30] S. Hollands and A. Ishibashi, "Asymptotic flatness at null infinity in higher dimensional gravity," hep-th/0311178.

[31] S. Hollands and R. M. Wald, "Conformal null infinity does not exist for radiating solutions in odd spacetime dimensions," Class. Quant. Grav. 21 (2004) 5139-5146, gr-qc/0407014.

[32] K. Tanabe, N. Tanahashi, and T. Shiromizu, "On asymptotic structure at null infinity in five dimensions," 0909.0426.

[33] L. Susskind, "Holography in the flat space limit," hep-th/9901079.

[34] J. Polchinski, "S-matrices from AdS spacetime," hep-th/9901076.

[35] J. de Boer and S. N. Solodukhin, "A holographic reduction of Minkowski space-time," Nucl. Phys. B665 (2003) 545-593, hep-th/0303006.

[36] G. Arcioni and C. Dappiaggi, "Exploring the holographic principle in asymptotically flat spacetimes via the BMS group," Nucl. Phys. B674 (2003) 553-592, hep-th/0306142.

[37] G. Arcioni and C. Dappiaggi, "Holography in asymptotically flat space-times and the BMS group," Class. Quant. Grav. 21 (2004) 5655, hep-th/0312186. 
[38] S. N. Solodukhin, "Reconstructing Minkowski space-time," hep-th/0405252.

[39] M. Gary and S. B. Giddings, "The flat space S-matrix from the AdS/CFT correspondence?,"0904.3544.

[40] T. Banks, "A critique of pure string theory: Heterodox opinions of diverse dimensions," hep-th/0306074

[41] C. Fefferman and C. Graham, Elie Cartan et les Mathématiques d'aujourd'hui, ch. Conformal Invariants, pp. 95-116. Astérisque, 1985.

[42] C. Graham and J. Lee, "Einstein metrics with prescribed conformal infinity on the ball," Adv. Math. 87 (1991) 186-225.

[43] M. Henningson and K. Skenderis, "The holographic Weyl anomaly," JHEP 07 (1998) 023, hep-th/9806087.

[44] M. Bañados, "Three-Dimensional Quantum Geometry and Black Holes," in Trends in Theoretical Physics II, H. Falomir, R. E. Gamboa Saravi, and F. A. Schaposnik, eds., vol. 484 of American Institute of Physics Conference Series, pp. 147-169. 1999.

[45] K. Skenderis and S. N. Solodukhin, "Quantum effective action from the AdS/CFT correspondence," Phys. Lett. B472 (2000) 316-322, hep-th/9910023.

[46] C. R. Graham, "Volume and area renormalizations for conformally compact Einstein metrics," math/9909042.

[47] C. Imbimbo, A. Schwimmer, S. Theisen, and S. Yankielowicz, "Diffeomorphisms and holographic anomalies," Class. Quant. Grav. 17 (2000) 1129-1138, hep-th/9910267.

[48] M. Rooman and P. Spindel, "Aspects of (2+1) dimensional gravity: AdS(3) asymptotic dynamics in the framework of Fefferman-Graham-Lee theorems," Annalen Phys. 9 (2000) 161-167, hep-th/9911142.

[49] K. Bautier, F. Englert, M. Rooman, and P. Spindel, "The Fefferman-Graham ambiguity and AdS black holes," Phys. Lett. B479 (2000) 291-298, hep-th/0002156.

[50] I. Papadimitriou and K. Skenderis, "Thermodynamics of asymptotically locally AdS spacetimes," JHEP 08 (2005) 004, hep-th/ 0505190.

[51] R. Penrose, "Asymptotic properties of fields and space-times," Phys. Rev. Lett. 10 (1963), no. 2, 66-68. 
[52] L. A. Tamburino and J. H. Winicour, "Gravitational fields in finite and conformal Bondi frames," Phys. Rev. 150 (1966) 1039.

[53] J. Winicour, "Logarithmic asymptotic flatness," Foundations of Physics 15 (05, 1985) 605-616.

[54] M. Bañados, C. Teitelboim, and J. Zanelli, "The black hole in three-dimensional space-time," Phys. Rev. Lett. 69 (1992) 1849-1851, hep-th/9204099.

[55] M. Bañados, M. Henneaux, C. Teitelboim, and J. Zanelli, "Geometry of the $(2+1)$ black hole," Phys. Rev. D48 (1993) 1506-1525, gr-qc/9302012.

[56] G. Barnich and F. Brandt, "Covariant theory of asymptotic symmetries, conservation laws and central charges," Nucl. Phys. B633 (2002) 3-82, hep-th/0111246.

[57] G. Barnich and G. Compère, "Surface charge algebra in gauge theories and thermodynamic integrability," J. Math. Phys. 49 (2008) 042901, 0708.2378.

[58] T. Regge and C. Teitelboim, "Role of surface integrals in the Hamiltonian formulation of general relativity," Ann. Phys. 88 (1974) 286.

[59] J. D. Brown and M. Henneaux, "On the Poisson brackets of differentiable generators in classical field theory," J. Math. Phys. 27 (1986) 489.

[60] E. T. Newman and R. Penrose, "Note on the Bondi-Metzner-Sachs Group," J. Math. Phys. 7 (1966) 863-870.

[61] A. A. Belavin, A. M. Polyakov, and A. B. Zamolodchikov, "Infinite conformal symmetry in two-dimensional quantum field theory," Nucl. Phys. B241 (1984) 333-380.

[62] R. Penrose, "Zero rest mass fields including gravitation: Asymptotic behavior," Proc. Roy. Soc. Lond. A284 (1965) 159.

[63] P. T. Chrusciel, M. A. H. MacCallum, and D. B. Singleton, "Gravitational waves in general relativity: 14. Bondi expansions and the polyhomogeneity of Scri," Proc. Roy. Soc. Lond. A436 (1992) 299-316, gr-qc/9305021.

[64] R. Geroch, "Asymptotic structure of space-time," in Symposium on the asymptotic structure of space-time, P. Esposito and L. Witten, eds., pp. 1-105. Plenum,New York, 1977.

[65] R. Wald, General Relativity. The University of Chicago Press, 1984. 
[66] E. Witten, “Talk given at Strings '98." available at http://online.kitp.ucsb.edu/online/strings98/witten/. 